\title{
Quantitative Heterodimerization of Aromatic Peptides through Host-Enhanced Polar-m Interactions for On-Resin Recognition
}

Xiaoyi Chen, Zehuan Huang, Guanglu Wu, Kamil Sokłolowski, Katherine King, Jade McCune, Oren Scherman

Submitted date: 04/05/2021 - Posted date: 07/05/2021

Licence: CC BY-NC-ND 4.0

Citation information: Chen, Xiaoyi; Huang, Zehuan; Wu, Guanglu; Sokłolowski, Kamil; King, Katherine; McCune, Jade; et al. (2021): Quantitative Heterodimerization of Aromatic Peptides through Host-Enhanced Polar- $\Pi$ Interactions for On-Resin Recognition. ChemRxiv. Preprint. https://doi.org/10.26434/chemrxiv.14538345.v1

Peptide dimerization plays an important role in both natural and artificial supramolecular systems. A major challenge to date is the quantitative heterodimerization of two peptides without formation of homodimers. Here, we employ a macrocyclic host to simultaneously encapsulate a canonical aromatic peptide and a non-canonical perfluorophenylalanine-containing peptide through polar- $\pi$ interactions, thus forming an unprecedented new series of heteropeptide dimers with high binding affinity. This new peptide heterodimerization was applied to on-resin recognition and separation of aromatic peptides in a peptide mixture exhibiting over $95 \%$ isolation purity. This research unveils a generic approach to exploit quantitative heteropeptide dimers for the design of supramolecular (bio)systems.

File list (2)

ESI.pdf (3.45 MiB)

view on ChemRxiv - download file

main text.pdf (1.32 MiB)

view on ChemRxiv - download file 


\title{
Supporting Information
}

\section{Quantitative Heterodimerization of Aromatic Peptides through Host-Enhanced Polar- $\pi$ Interactions for On-Resin Recognition}

\author{
Xiaoyi Chen, ${ }^{\dagger}$ Zehuan Huang, ${ }^{\dagger}$ Guanglu Wu, Kamil Sokłolowski, \\ Katherine King, Jade A. McCune, and Oren A. Scherman* \\ $\dagger$ These authors contributed equally to this work \\ Melville Laboratory for Polymer Synthesis, Yusuf Hamied Department of Chemistry, University of Cambridge, \\ Lensfield Road, Cambridge, CB2 1EW, United Kingdom \\ E-mail: oas23@cam.ac.uk
}

\section{Table of Contents}

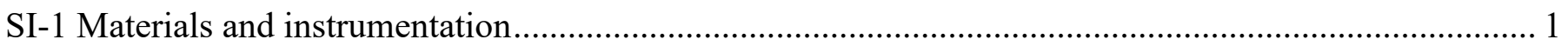

SI-2 Synthesis and characterization of model peptides .............................................................. 4

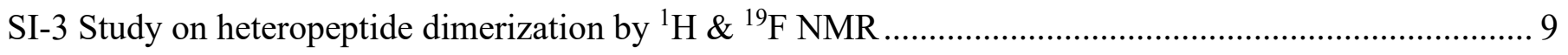

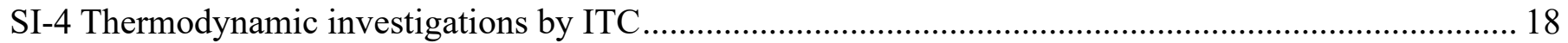

SI-5 Quantification of on-resin recognition by UV-Vis \& ${ }^{1} \mathrm{H}$ NMR ................................................... 24

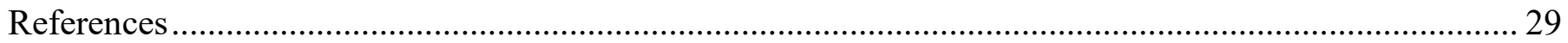




\section{SI-1 Materials and instrumentation}

Materials. Unless noted, all the starting materials were purchased from commercial suppliers and were used without further purification: (L)-Fmoc-Glu(OtBu)-OH, (L)-Fmoc-Gly-OH, (L)-Fmoc-Leu-OH, (L)-FmocLys(Boc)-OH， (L)-Fmoc-Phe-OH， (L)-Fmoc-Trp(Boc)-OH， (L)-Fmoc-Tyr(tBu)-OH， (L)-Fmoc-3-(2naphthyl)-alanine-OH, (L)-Fmoc-perfluorophenylalanine-OH, piperidine, diisopropylethylamine (DIPEA), triisopropylsilane (TIPS), trifluoroacetic acid (TFA), diethyl ether, N,N,N',N'-Tetramethyl-O-(1Hbenzotriazol-1-yl)uronium hexafluorophosphate, dimethylformamide (DMF), dichloromethane, monobasic sodium phosphate $\left(\mathrm{NaH}_{2} \mathrm{PO}_{4}\right)$, dibasic sodium phosphate $\left(\mathrm{Na}_{2} \mathrm{HPO}_{4}\right)$, deuterium oxide $\left(\mathrm{D}_{2} \mathrm{O}, \mathrm{D}, 99.8 \%\right)$, 1,3-dimethylaminoadamantane hydrochloride (memantine hydrochloride, DMADA), Rink Amide-AM Resin, H-Rink Amide ChemMatrix Resin. Phosphate buffer solution was prepared by adding $10 \mathrm{mM}$ $\mathrm{NaH}_{2} \mathrm{PO}_{4}$ solution to $10 \mathrm{mM} \mathrm{Na} \mathrm{HPO}_{4}$ solution until $\mathrm{pH}$ equal to 7.0. Cucurbit[8] uril (CB[8]) was synthesized and purified according to a previous report, ${ }^{[1]}$ and the molecular weight for $\mathrm{CB}[8]$ was calibrated as $1600 \mathrm{~g} / \mathrm{mol}$. Water was obtained from a Milli-Q Integral Water Purification System $(22.5 \mathrm{M} \Omega \cdot \mathrm{cm})$.

Peptide Synthesis \& Purification. All of the tripeptides (XGG, GXG, GGX) were synthesized on an automated microwave peptide synthesizer (Liberty Blue, CEM) using standard 9-fluorenylmethoxycarbonyl (Fmoc) chemistry. Peptides were cleaved from the Rink Amide-AM resin with a mixture of 95\% TFA, 2.5\% TIPS, and $2.5 \% \mathrm{H}_{2} \mathrm{O}$ and shaken for $1 \mathrm{hr}$ at room temperature. The resin was filtered and washed with the cleavage mixture. After evaporating most of the cleavage mixture, the peptides were crushed out and washed three times with cold diethyl ether. The crude peptides were purified using reverse-phase high performance

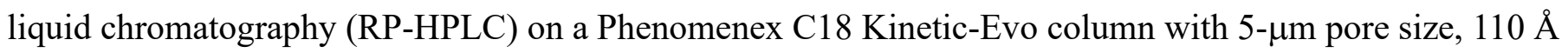
particle size and $150 \times 21.2 \mathrm{~mm}$ dimensions. The mobile phase for water contained $0.1 \%$ TFA. The purification method gradient was set from 5\% acetonitrile and 95\% water to $100 \%$ acetonitrile. Dry peptides were obtained (as TFA salt) by lyophilization and then verified by NMR and ESI-MS. On resin peptide F'GGGGG were synthesized using H-Rink Amide ChemMatrix Resin using the same method above. The F'GGGGG-functionalized resin was washed with DMF for 3 times and then left in $\mathrm{D}_{2} \mathrm{O}$ (for 3 days, change with fresh $\mathrm{D}_{2} \mathrm{O}$ each day). Then, the washed F'GGGGG resin was preserved in $\mathrm{D}_{2} \mathrm{O}$ in fridge at $277 \mathrm{~K}$.

Nuclear Magnetic Resonance Spectroscopy (NMR). ${ }^{1} \mathrm{H},{ }^{13} \mathrm{C} \&{ }^{19} \mathrm{~F}$ NMR spectra of pure peptides were acquired in $\mathrm{D}_{2} \mathrm{O}$ at $298 \mathrm{~K}$ on a Bruker AVANCE $400(400 \mathrm{MHz})$ apparatus and a Bruker AVANCE 500 apparatus with TCI Cryoprobe (500 MHz). ${ }^{1} \mathrm{H} \&{ }^{19} \mathrm{~F}$ NMR spectra of other experiments (NMR titration, onresin binding test) were acquired in phosphate buffer $\left(\mathrm{D}_{2} \mathrm{O}\right)$ with $\mathrm{pH} 7.0$ on a Bruker AVANCE 400 (400 $\mathrm{MHz}$ ) apparatus. Chemical shifts were referenced to the residual solvent peak of HDO at $4.79 \mathrm{ppm}$. A standard procedure of NMR quantification for on-resin recognition within a peptide mixture is shown:

A solution of mixed peptides containing WGG, KGG, LGG, EGG, CB[8] $([\mathrm{XGG}]=\mathrm{CB}[8]=1 \mathrm{mM})$ was used as the original cycle 0 solution. For cycle $1,1.0 \mathrm{ml}$ of the mixed peptides $(1 \mathrm{mM})$ was added to the F'GGGGG-functionalized resin $(10 \mathrm{mM})$ and shaken for $10 \mathrm{~min}$ at room temperature. After draining, $1 \mathrm{ml}$ of $2 \mathrm{mM}$ DMADA solution was added, shaken for $45 \mathrm{~min}$, and collected as the released solution. For cycle 
2, fresh $\mathrm{CB}$ [8] (2.88 $\mathrm{mg}, 1.8 \mu \mathrm{mol}$ ) was added to $0.9 \mathrm{ml}$ of the released solution (2 mM DMADA, $1.8 \mu \mathrm{mol}$ ) from cycle 1 to bind with excess DMADA and the isolated WGG. Around $0.1 \mathrm{ml}$ solution was lost after each cycle. This new solution was added with the resin, shaken for $45 \mathrm{~min}$, drained and then $0.9 \mathrm{ml}$ of $2 \mathrm{mM}$ DMADA solution was added to generate the released solution from cycle 2. For cycle 3, fresh CB[8] (2.56 $\mathrm{mg}, 1.6 \mu \mathrm{mol}$ ) was added to $0.8 \mathrm{ml}$ of the released solution ( $2 \mathrm{mM}$ DMADA, $1.6 \mu \mathrm{mol}$ ) from cycle 2 . This new solution was added with the resin, shaken for $45 \mathrm{~min}$, drained and then $0.8 \mathrm{ml}$ of DMADA solution was added to generate the released solution from cycle 3 . The original cycle 0 solution and the subsequent released solutions from each cycle were tested with NMR. After NMR analysis, the relative amount of WGG was determined by integrating a set of doublet peak at $3.36 \mathrm{ppm}$; the relative amount of KGG was determined by integrating a set of triplet peak at $3.02 \mathrm{ppm}$; the relative amount of EGG was determined by integrating a set of triplet peak at $2.37 \mathrm{ppm}$. There was a singlet peak of KGG, LGG, EGG that overlapped at $3.95 \mathrm{ppm}$. Thus, the relative amount of LGG was determined by the relative amount of KGG and EGG subtracted from the integration of peak at $3.95 \mathrm{ppm}$.

Isothermal Titration Calorimetry (ITC). ITC experiments were operated on a Malvern MicroCal AutoITC200 apparatus at $298 \mathrm{~K}$ in $10 \mathrm{mM}$ phosphate buffer with $\mathrm{pH}$ 7.0. In a typical experiment for heteroternary complexation, the 1:1 mixture of $\mathrm{CB}[8]$ and F' GG was loaded in the sample cell with a concentration of 0.2 $\mathrm{mM}$, and the second guest peptide was loaded in the syringe with a concentration of $3.0 \mathrm{mM}$. One titration experiment consisted of 1 injection of $0.6 \mu \mathrm{L}$ and 32 consecutive injections of $1.2 \mu \mathrm{L}$ with 90 s intervals between injections. The first data point was removed before analysis. The ITC data were fitted by MicroCal Analysis Centre software using one set of sites model. In a typical experiment for homoternary complexation, the host molecule (CB[8]) was loaded in the sample cell with a concentration of $0.1 \mathrm{mM}$, and the guest peptide was loaded in the syringe with a concentration of $2.0 \mathrm{mM}$. The injection and interval settings were the same as above. The first data point was removed from the data set before analysis. The obtained ITC curves were fitted by MicroCal Analysis Centre software using sequential binding model.

Electrospray Ionization Mass Spectrometry (ESI-MS). ESI-MS data were obtained on a Thermo Fisher Q Exactive Orbitrap mass spectrometer with a nanospraying ion source. Positive mode was chosen for all experiments at a working temperature of $320^{\circ} \mathrm{C}$ and a capillary voltage of $1.5 \mathrm{kV}$. All the solutions for ESIMS were prepared in pure water.

UV-Vis Spectrometry (UV-Vis). UV-Vis spectra were obtained on a Cary 4000 Ultraviolet-visible Spectrometer. A pair of quartz cuvettes with $2 \mathrm{~mm}$ gap was used. Double beam mode with a fixed $2 \mathrm{~nm}$ spectral bandwidth and baseline correction were selected. All spectra were recorded with 3 repeats. In Figure 4b, WGG-CB[8] original, resin-treated, released and control group WGG original, and resin-treated solutions were tested on a UV-Vis spectrometer. In a typical run, $300 \mu \mathrm{l}$ of $1 \mathrm{mM}$ solution was transferred to the quartz cuvette and was scanned comparing to phosphate buffer in $\mathrm{D}_{2} \mathrm{O}$. In Figure $4 \mathrm{c}$, similar tests were done as Figure $4 \mathrm{~b}$ for the series of resin-treated and released solutions. A standard procedure of UV quantification for on-resin recognition is shown as follows: 
Using a model tripeptide WGG as an example, $10 \mu$ mol F'GGGGG equivalent of resin $\mathrm{D}_{2} \mathrm{O}$ suspension was added to a $12 \mathrm{ml}$ syringe with a polyester frit $(\mathrm{r}=8.0 \mathrm{~mm}, \mathrm{~h}=2.0 \mathrm{~mm})$. After $\mathrm{D}_{2} \mathrm{O}$ was drained, $1 \mathrm{ml}$ of $1 \mathrm{mM}$ WGG:CB$[8]=1: 1$ solution (original solution, in $\mathrm{D}_{2} \mathrm{O}$, phosphate buffer, $\mathrm{pH}=7.0$ ) was added to the resin. Then, the plunge and a stopper were assembled to the syringe to form a sealed chamber. The syringe was transferred onto a wrist-action shaker and shaken for $10 \mathrm{~min}$. Then, the liquid (resin-treated solution) in syringe was drained thoroughly and the resin was washed with $\mathrm{D}_{2} \mathrm{O}$ once. After that, $1 \mathrm{ml}$ of $2 \mathrm{mM}$ DMADA solution (in $\mathrm{D}_{2} \mathrm{O}$, phosphate buffer, $\mathrm{pH}=7.0$ ) was added to the resin and shaken for an additional $45 \mathrm{~min}$. The solution (released solution) was drained for further testing. For efficiency test over 3 cycles (Figure 4c), WGG:CB $[8]=1: 1$ solution and DMADA solution were alternatively added to resin, shaken and drained for three cycles and produced a series of resin-treated and released solutions. 
SI-2 Synthesis and characterization of model peptides<smiles>NC(=O)CNC(=O)CNC(=O)[C@@H](Cc1ccccc1)Cc1c(F)c(F)c(F)c(F)c1F</smiles><smiles>NC(=O)CNC(=O)CNC(=O)[C@H](N)Cc1c[nH]c2ccccc12</smiles><smiles>NC(=O)CNC(=O)CNC(=O)[C@H](N)Cc1ccccc1</smiles>

FGG<smiles>NC(=O)CNC(=O)CNC(=O)[C@H](N)Cc1ccc(O)cc1</smiles><smiles>NC(=O)CNC(=O)CNC(=O)[C@H](Cc1ccc2ccccc2c1)Nc1ccccc1</smiles><smiles>NCC(=O)NC(Cc1c(F)c(F)c(F)c(F)c1F)C(=O)NCC(N)=O</smiles>
GF'G<smiles>NCC(=O)NC(Cc1c[nH]c2ccccc12)C(=O)NCC(N)=O</smiles>

GWG<smiles>NCC(=O)NC(Cc1ccccc1)C(=O)NCC(N)=O</smiles><smiles>NCC(=O)NC(Cc1ccc(O)cc1)C(=O)NCC(N)=O</smiles><smiles>NCC(=O)NC(Cc1ccc2ccccc2c1)C(=O)NCC(N)=O</smiles>
GNpG

(continue on next page)<smiles>NCC(=O)NCC(=O)N[C@@H](Cc1c(F)c(F)c(F)c(F)c1F)C(N)=O</smiles><smiles>NCC(=O)NCC(=O)N[C@@H](Cc1c[nH]c2ccccc12)C(N)=O</smiles><smiles>NCC(=O)NCC(=O)N[C@@H](Cc1ccccc1)C(N)=O</smiles><smiles>NCC(=O)NCC(=O)N[C@@H](Cc1ccc(O)cc1)C(N)=O</smiles><smiles>NCC(=O)NCC(=O)N[C@@H](Cc1ccc2ccccc2c1)C(N)=O</smiles> 
(continued)
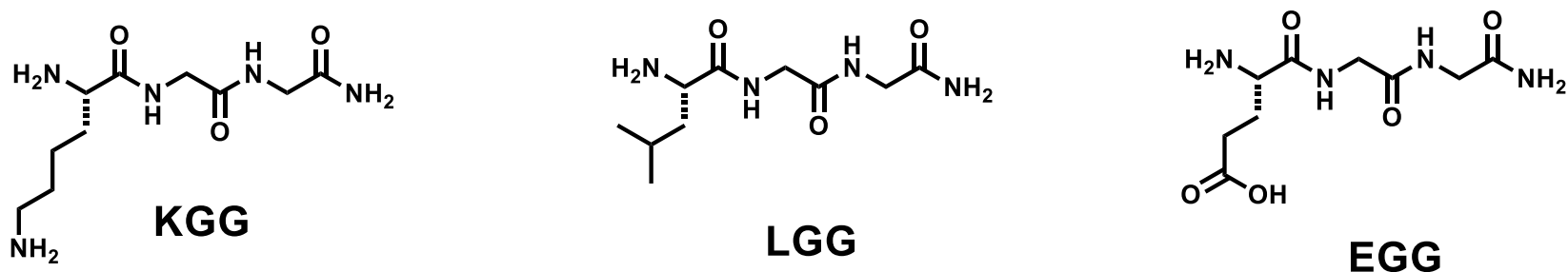

EGG

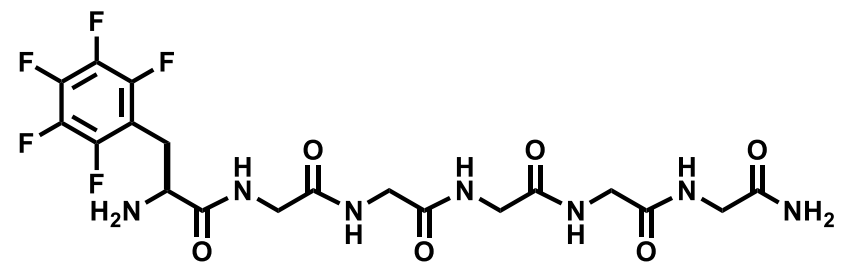

F'GGGGG

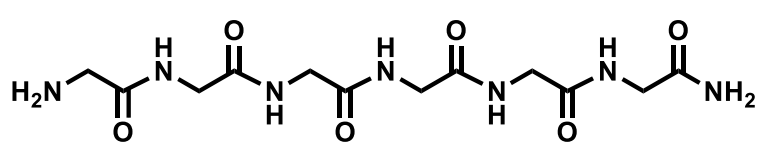

GGGGGG

Chart S1 Molecular structures of all peptides used in this study. All these peptides are TFA salts.

(S)-2-amino-N-(2-((2-amino-2-oxoethyl)amino)-2-oxoethyl)-3-(perfluorophenyl)propenamide (F'GG) ${ }^{1} \mathrm{H}$ NMR (500 MHz, D $\left.2 \mathrm{O}, \mathrm{ppm}\right): \delta=4.32(\mathrm{t}, 1 \mathrm{H}), 3.99(\mathrm{q}, 2 \mathrm{H}), 3.93(\mathrm{~s}, 2 \mathrm{H}), 3.37(\mathrm{~d}, 2 \mathrm{H}) ;{ }^{13} \mathrm{C}$ NMR $(125$ $\left.\mathrm{MHz}, \mathrm{D}_{2} \mathrm{O}\right): \delta=174.01,170.89,168.81,146.39,144.44,141.90,139.89,138.49,136.49,107.29,52.04$, 42.30, 41.89, 23.85; ${ }^{19} \mathrm{~F}$ NMR (375 MHz, $\left.\mathrm{D}_{2} \mathrm{O}, \mathrm{ppm}\right): \delta=-142.65,-154.41,-162.18$; ESI MS for [M-H] ${ }^{+}$: calc. $\mathrm{m} / \mathrm{z}=369.10$, found $\mathrm{m} / \mathrm{z}=369.17$.

(S)-N-(2-amino-2-oxoethyl)-2-(2-aminoacetamido)-3-(perfluorophenyl)propanamide (GF'G) ${ }^{1} \mathrm{H}$ NMR $\left(500 \mathrm{MHz}, \mathrm{D}_{2} \mathrm{O}, \mathrm{ppm}\right): \delta=4.77-4.74(\mathrm{~m}, 1 \mathrm{H}), 3.95-3.86(\mathrm{~m}, 2 \mathrm{H}), 3.85-3.79(\mathrm{~m}, 2 \mathrm{H}), 3.32(\mathrm{dd}, 1 \mathrm{H})$, $3.20(\mathrm{dd}, 1 \mathrm{H}) ;{ }^{13} \mathrm{C}$ NMR $\left(125 \mathrm{MHz}, \mathrm{D}_{2} \mathrm{O}, \mathrm{ppm}\right): \delta=173.56,172.10,166.91,146.25,144.32,141.27,139.28$, 138.29, 136.33, 109.48, 52.55, 42.00, 40.22, 24.43; $\left.{ }^{19} \mathrm{~F} \mathrm{NMR} \mathrm{(375} \mathrm{MHz,} \mathrm{D}_{2} \mathrm{O}, \mathrm{ppm}\right): \delta=-143.48,-155.99$, -162.89; ESI MS for $[\mathrm{M}-\mathrm{H}]^{+}$: calc. $\mathrm{m} / \mathrm{z}=369.10$, found $\mathrm{m} / \mathrm{z}=369.17$.

(S)-2-(2-(2-aminoacetamido)acetamido)-3-(perfluorophenyl)propanamide (GGF') ${ }^{1} \mathrm{H}$ NMR (500 MHz, $\left.\mathrm{D}_{2} \mathrm{O}, \mathrm{ppm}\right): \delta=4.73-4.70(\mathrm{~m}, 1 \mathrm{H}), 3.96(\mathrm{~s}, 2 \mathrm{H}), 3.88(\mathrm{~s}, 2 \mathrm{H}), 3.31(\mathrm{dd}, 1 \mathrm{H}), 3.16(\mathrm{dd}, 1 \mathrm{H}) ;{ }^{13} \mathrm{C} \mathrm{NMR}(125$ $\left.\mathrm{MHz}, \mathrm{D}_{2} \mathrm{O}, \mathrm{ppm}\right): \delta=174.32,170.86,167.64,146.27,144.34,141.17,139.17,138.24,136.27,109.89,52.04$, 42.05, 40.28, 24.52; ${ }^{19} \mathrm{~F}$ NMR (375 MHz, $\left.\mathrm{D}_{2} \mathrm{O}, \mathrm{ppm}\right): \delta=-143.47,-156.31,-163.04$; ESI MS for $[\mathrm{M}-\mathrm{H}]^{+}$: calc. $\mathrm{m} / \mathrm{z}=369.10$, found $\mathrm{m} / \mathrm{z}=369.17$.

(S)-2-amino-N-(2-((2-amino-2-oxoethyl)amino)-2-oxoethyl)-3-(1H-indol-3-yl)propanamide (WGG) ${ }^{1} \mathrm{H}$ NMR (500 MHz, D $2 \mathrm{O}, \mathrm{ppm}): \delta=7.64(\mathrm{~d}, 1 \mathrm{H}), 7.54(\mathrm{~d}, 1 \mathrm{H}), 7.35(\mathrm{~s}, 1 \mathrm{H}), 7.29(\mathrm{t}, 1 \mathrm{H}), 7.21(\mathrm{t}, 1 \mathrm{H}), 4.36(\mathrm{t}$, $1 \mathrm{H}), 3.92(\mathrm{~d}, 1 \mathrm{H}), 3.91-3.75(\mathrm{~m}, 3 \mathrm{H}), 3.49-3.38(\mathrm{~m}, 2 \mathrm{H}) ;{ }^{13} \mathrm{C} \mathrm{NMR}\left(125 \mathrm{MHz}, \mathrm{D}_{2} \mathrm{O}, \mathrm{ppm}\right): \delta=173.90$, 171.22, 170.24, 136.11, 126.42, 125.23, 122.21, 119.61, 117.98, 112.03, 106.24, 53.68, 42.36, 41.83, 26.70; ESI MS for $[\mathrm{M}-\mathrm{H}]^{+}$: calc. $\mathrm{m} / \mathrm{z}=318.16$, found $\mathrm{m} / \mathrm{z}=318.34$. 
(S)-N-(2-amino-2-oxoethyl)-2-(2-aminoacetamido)-3-(1H-indol-3-yl)propanamide (GWG) ${ }^{1} \mathrm{H}$ NMR $\left(500 \mathrm{MHz}, \mathrm{D}_{2} \mathrm{O}, \mathrm{ppm}\right): \delta=7.67(\mathrm{~d}, 1 \mathrm{H}), 7.52(\mathrm{~d}, 1 \mathrm{H}), 7.30-7.24(\mathrm{~m}, 2 \mathrm{H}), 7.19(\mathrm{t}, 1 \mathrm{H}), 4.77-4.69(\mathrm{~m}, 1 \mathrm{H})$, $3.84(\mathrm{~d}, 1 \mathrm{H}), 3.81-3.73(\mathrm{~m}, 2 \mathrm{H}), 3.70(\mathrm{~d}, 1 \mathrm{H}), 3.36-3.23(\mathrm{~m}, 2 \mathrm{H}) ;{ }^{13} \mathrm{C}$ NMR $\left(125 \mathrm{MHz}, \mathrm{D}_{2} \mathrm{O}, \mathrm{ppm}\right): \delta=$ 173.90, 173.77, 166.95, 136.04, 126.64, 124.54, 122.00, 119.38, 118.25, 111.89, 108.60, 54.89, 42.00, 40.23, 26.96; ESI MS for $[\mathrm{M}-\mathrm{H}]^{+}$: calc. $\mathrm{m} / \mathrm{z}=318.16$, found $\mathrm{m} / \mathrm{z}=318.34$.

(S)-2-(2-(2-aminoacetamido)acetamido)-3-(1H-indol-2-yl)propanamide (GGW) ${ }^{1} \mathrm{H}$ NMR (500 $\mathrm{MHz}$, $\left.\mathrm{D}_{2} \mathrm{O}, \mathrm{ppm}\right): \delta=7.70(\mathrm{~d}, 1 \mathrm{H}), 7.52(\mathrm{~d}, 1 \mathrm{H}), 7.30-7.24(\mathrm{~m}, 2 \mathrm{H}), 7.19(\mathrm{t}, 1 \mathrm{H}), 4.68(\mathrm{dd}, 1 \mathrm{H}), 3.99-3.87(\mathrm{~m}$, 2H), 3.77 (s, 2H), 3.34 (dd, 1H), 3.23 (dd, 1H); ${ }^{13} \mathrm{C}$ NMR (125 MHz, $\left.\mathrm{D}_{2} \mathrm{O}, \mathrm{ppm}\right): \delta=176.11,170.80,167.56$, $136.05,126.84,124.49,121.89,119.30,118.29,111.84,108.86,54.13,42.11,40.23,26.96$; ESI MS for [M$\mathrm{H}]^{+}$: calc. $\mathrm{m} / \mathrm{z}=318.16$, found $\mathrm{m} / \mathrm{z}=318.34$.

(S)-2-amino-N-(2-((2-amino-2-oxoethyl)amino)-2-oxoethyl)-3-phenylpropanamide (FGG) ${ }^{1} \mathrm{H}$ NMR $\left(500 \mathrm{MHz}, \mathrm{D}_{2} \mathrm{O}, \mathrm{ppm}\right): \delta=7.47-7.36(\mathrm{~m}, 3 \mathrm{H}), 7.35-7.29(\mathrm{~m}, 2 \mathrm{H}), 4.32(\mathrm{t} 1 \mathrm{H}), 4.01(\mathrm{~d}, 1 \mathrm{H}), 3.94-3.87$ $(\mathrm{m}, 3 \mathrm{H}), 3.29-3.20(\mathrm{~m}, 2 \mathrm{H}) ;{ }^{13} \mathrm{C}$ NMR (125 MHz, $\left.\mathrm{D}_{2} \mathrm{O}, \mathrm{ppm}\right): \delta=173.97,171.27,169.82,133.68,129.35$, 129.14, 128.00, 54.41, 42.26, 41.90, 36.69; ESI MS for $[\mathrm{M}-\mathrm{H}]^{+}:$calc. $\mathrm{m} / \mathrm{z}=279.15$, found $\mathrm{m} / \mathrm{z}=279.34$.

(S)-N-(2-amino-2-oxoethyl)-2-(2-aminoacetamido)-3-phenylpropanamide (GFG) ${ }^{1} \mathrm{H}$ NMR (500 MHz, $\left.\mathrm{D}_{2} \mathrm{O}, \mathrm{ppm}\right): \delta=7.44-7.37(\mathrm{~m}, 2 \mathrm{H}), 7.37-7.31(\mathrm{~m}, 1 \mathrm{H}), 7.34-7.28(\mathrm{~m}, 2 \mathrm{H}), 4.68(\mathrm{t}, 1 \mathrm{H}), 3.86(\mathrm{t}, 2 \mathrm{H})$, $3.81-3.74(\mathrm{~m}, 2 \mathrm{H}), 3.16(\mathrm{dd}, 1 \mathrm{H}), 3.07(\mathrm{dd}, 1 \mathrm{H}) ;{ }^{13} \mathrm{C}$ NMR $\left(125 \mathrm{MHz}, \mathrm{D}_{2} \mathrm{O}, \mathrm{ppm}\right): \delta=173.76,173.49$, 166.96, 136.05, 129.14, 128.80, 127.28, 55.32, 41.99, 40.18, 36.93; ESI MS for $[\mathrm{M}-\mathrm{H}]^{+}: \mathrm{calc} . \mathrm{m} / \mathrm{z}=279.15$, found $\mathrm{m} / \mathrm{z}=279.34$.

(S)-2-(2-(2-aminoacetamido)acetamido)-3-phenylpropanamide (GGF) ${ }^{1} \mathrm{H}$ NMR (500 MHz, $\mathrm{D}_{2} \mathrm{O}$, ppm): $\delta=7.43-7.36(\mathrm{~m}, 2 \mathrm{H}), 7.36-7.29(\mathrm{~m}, 2 \mathrm{H}), 7.30(\mathrm{t}, 1 \mathrm{H}), 4.62(\mathrm{dd}, 1 \mathrm{H}), 3.94(\mathrm{q}, 2 \mathrm{H}), 3.85(\mathrm{~s}, 2 \mathrm{H}), 3.19(\mathrm{dd}$, $1 \mathrm{H}), 3.01(\mathrm{dd}, 1 \mathrm{H}) ;{ }^{13} \mathrm{C}$ NMR $\left(125 \mathrm{MHz}, \mathrm{D}_{2} \mathrm{O}, \mathrm{ppm}\right): \delta=175.70,170.82,167.57,136.41,129.14,128.69$, 127.11, 54.68, 42.01, 40.29, 36.98; ESI MS for $[\mathrm{M}-\mathrm{H}]^{+}:$calc. $\mathrm{m} / \mathrm{z}=279.15$, found $\mathrm{m} / \mathrm{z}=279.34$.

(S)-2-amino-N-(2-((2-amino-2-oxoethyl)amino)-2-oxoethyl)-3-(4-hydroxyphenyl)propanamide (YGG) ${ }^{1} \mathrm{H}$ NMR $\left(500 \mathrm{MHz}, \mathrm{D}_{2} \mathrm{O}, \mathrm{ppm}\right): \delta=7.22-7.15(\mathrm{~m}, 2 \mathrm{H}), 6.93-6.87(\mathrm{~m}, 2 \mathrm{H}), 4.26(\mathrm{t}, 1 \mathrm{H}), 4.05-3.97(\mathrm{~m}$, $1 \mathrm{H}), 3.96-3.88(\mathrm{~m}, 3 \mathrm{H}), 3.16(\mathrm{~d}, 2 \mathrm{H}) ;{ }^{13} \mathrm{C} \mathrm{NMR}\left(125 \mathrm{MHz}, \mathrm{D}_{2} \mathrm{O}, \mathrm{ppm}\right): \delta=173.94,171.28,169.89,155.13$, $130.78,125.41,115.81,54.49,42.26,41.89,35.86$; ESI MS for $[M-H]^{+}:$calc. $\mathrm{m} / \mathrm{z}=295.14$, found $\mathrm{m} / \mathrm{z}=$ 295.34 .

(S)-N-(2-amino-2-oxoethyl)-2-(2-aminoacetamido)-3-(4-hydroxyphenyl)propanamide (GYG) ${ }^{1} \mathrm{H}$ NMR $\left(500 \mathrm{MHz}, \mathrm{D}_{2} \mathrm{O}, \mathrm{ppm}\right): \delta=7.20-7.14(\mathrm{~m}, 2 \mathrm{H}), 6.87(\mathrm{~m}, 2 \mathrm{H}), 4.61(\mathrm{t}, 1 \mathrm{H}), 3.90-3.83(\mathrm{~m}, 1 \mathrm{H}), 3.87-3.71$ $(\mathrm{m}, 3 \mathrm{H}), 3.07(\mathrm{dd}, 1 \mathrm{H}), 3.00(\mathrm{dd}, 1 \mathrm{H}) ;{ }^{13} \mathrm{C} \mathrm{NMR}\left(125 \mathrm{MHz}, \mathrm{D}_{2} \mathrm{O}, \mathrm{ppm}\right): \delta=173.79,173.59,166.94,154.52$, 130.50, 127.82, 115.48, 55.51, 41.98, 40.19, 36.12; ESI MS for $[\mathrm{M}-\mathrm{H}]^{+}$: calc. $\mathrm{m} / \mathrm{z}=295.14$, found $\mathrm{m} / \mathrm{z}=$ 295.25 . 
(S)-2-(2-(2-aminoacetamido)acetamido)-3-(4-hydroxyphenyl)propanamide (GGY) ${ }^{1} \mathrm{H}$ NMR (500 MHz, $\left.\mathrm{D}_{2} \mathrm{O}, \mathrm{ppm}\right): \delta=7.20-7.13(\mathrm{~m}, 2 \mathrm{H}), 6.90-6.83(\mathrm{~m}, 2 \mathrm{H}), 4.56(\mathrm{dd}, 1 \mathrm{H}), 3.95(\mathrm{q}, 2 \mathrm{H}), 3.86(\mathrm{~s}, 2 \mathrm{H}), 3.10(\mathrm{dd}$, 1H), $2.94(\mathrm{dd}, 1 \mathrm{H}) ;{ }^{13} \mathrm{C}$ NMR $\left(125 \mathrm{MHz}, \mathrm{D}_{2} \mathrm{O}, \mathrm{ppm}\right): \delta=175.77,170.79,167.57,154.37,130.49,128.22$, 115.38, 54.84, 42.01, 40.30, 36.18; ESI MS for $[\mathrm{M}-\mathrm{H}]^{+}:$calc. $\mathrm{m} / \mathrm{z}=295.14$, found $\mathrm{m} / \mathrm{z}=295.34$.

(S)-2-amino-N-(2-((2-amino-2-oxoethyl)amino)-2-oxoethyl)-3-(naphthalen-2-yl)propanamide (NpGG) ${ }^{1} \mathrm{H}$ NMR (500 MHz, $\left.\mathrm{D}_{2} \mathrm{O}, \mathrm{ppm}\right): \delta=7.97(\mathrm{t}, 2 \mathrm{H}), 7.95-7.90(\mathrm{~m}, 1 \mathrm{H}), 7.83-7.79(\mathrm{~m}, 1 \mathrm{H}), 7.64-7.55(\mathrm{~m}$, 2H), $7.46(\mathrm{dd}, 1 \mathrm{H}), 4.44-4.39(\mathrm{~m}, 1 \mathrm{H}), 3.89(\mathrm{~s}, 2 \mathrm{H}), 3.74-3.62(\mathrm{~m}, 2 \mathrm{H}), 3.44(\mathrm{dd}, 1 \mathrm{H}), 3.37(\mathrm{dd}, 1 \mathrm{H}) ;{ }^{13} \mathrm{C}$ NMR (125 MHz, $\left.\mathrm{D}_{2} \mathrm{O}, \mathrm{ppm}\right): \delta=173.74,171.04,169.72,133.04,132.38,131.40,128.84,128.33,127.72$, $127.62,126.92,126.84,126.61,54.27,42.23,41.68,36.87$; ESI MS for $[\mathrm{M}-\mathrm{H}]^{+}:$calc. $\mathrm{m} / \mathrm{z}=329.16$, found $\mathrm{m} / \mathrm{z}=329.34$.

(S)-N-(2-amino-2-oxoethyl)-2-(2-aminoacetamido)-3-(naphthalen-2-yl)propanamide (GNpG) ${ }^{1} \mathrm{H}$ NMR $\left(500 \mathrm{MHz}, \mathrm{D}_{2} \mathrm{O}, \mathrm{ppm}\right): \delta=7.93(\mathrm{td}, 3 \mathrm{H}), 7.80-7.76(\mathrm{~m}, 1 \mathrm{H}), 7.57(\mathrm{tt}, 2 \mathrm{H}), 7.46(\mathrm{dd}, 1 \mathrm{H}), 4.77-4.74(\mathrm{~m}$, $1 \mathrm{H}), 3.84(\mathrm{dd}, 2 \mathrm{H}), 3.73(\mathrm{dd}, 2 \mathrm{H}), 3.32(\mathrm{dd}, 1 \mathrm{H}), 3.23(\mathrm{dd}, 1 \mathrm{H}) ;{ }^{13} \mathrm{C} \mathrm{NMR}\left(125 \mathrm{MHz}, \mathrm{D}_{2} \mathrm{O}, \mathrm{ppm}\right): \delta=173.67$, $173.45,166.94,133.76,133.04,132.10,128.31,127.75,127.62,127.47,127.24,126.62,126.21,55.25$, 41.96, 40.16, 37.13; ESI MS for $[\mathrm{M}-\mathrm{H}]^{+}:$calc. $\mathrm{m} / \mathrm{z}=329.16$, found $\mathrm{m} / \mathrm{z}=329.34$.

(S)-2-(2-(2-aminoacetamido)acetamido)-3-(naphthalen-2-yl)propanamide (GGNp) ${ }^{1} \mathrm{H}$ NMR (500 MHz, $\left.\mathrm{D}_{2} \mathrm{O}, \mathrm{ppm}\right): \delta=7.97-7.89(\mathrm{~m}, 3 \mathrm{H}), 7.79-7.75(\mathrm{~m}, 1 \mathrm{H}), 7.57(\mathrm{tt}, 2 \mathrm{H}), 7.46(\mathrm{dd}, 1 \mathrm{H}), 4.73(\mathrm{dd}, 1 \mathrm{H}), 3.90$ (q, 2H), $3.76(\mathrm{~s}, 2 \mathrm{H}), 3.36(\mathrm{dd}, 1 \mathrm{H}), 3.16(\mathrm{dd}, 1 \mathrm{H}) ;{ }^{13} \mathrm{C} \mathrm{NMR}\left(125 \mathrm{MHz}, \mathrm{D}_{2} \mathrm{O}, \mathrm{ppm}\right): \delta=175.66,170.78$, 167.47, 134.17, 133.03, 132.06, 128.19, 127.71, 127.61, 127.49, 127.31, 126.51, 126.11, 54.53, 42.02, 40.21, 37.16; ESI MS for $[\mathrm{M}-\mathrm{H}]^{+}$: calc. $\mathrm{m} / \mathrm{z}=329.16$, found $\mathrm{m} / \mathrm{z}=329.34$.

(S)-2,6-diamino-N-(2-((2-amino-2-oxoethyl)amino)-2-oxoethyl)hexanamide (KGG) ${ }^{1} \mathrm{H}$ NMR (500 MHz, $\left.\mathrm{D}_{2} \mathrm{O}, \mathrm{ppm}\right): \delta=4.12-4.02(\mathrm{~m}, 3 \mathrm{H}), 3.95(\mathrm{~s}, 2 \mathrm{H}), 3.02(\mathrm{t}, 2 \mathrm{H}), 2.01-1.90(\mathrm{~m}, 2 \mathrm{H}), 1.73(\mathrm{p}, 2 \mathrm{H}), 1.55-1.43$ $(\mathrm{m}, 2 \mathrm{H}) ;{ }^{13} \mathrm{C}$ NMR $\left(125 \mathrm{MHz}, \mathrm{D}_{2} \mathrm{O}, \mathrm{ppm}\right): \delta=174.05,171.36,170.30,52.94,42.19,41.93,38.93,30.18$, 26.29, 21.06; ESI MS for $[\mathrm{M}-\mathrm{H}]^{+}:$calc. $\mathrm{m} / \mathrm{z}=260.17$, found $\mathrm{m} / \mathrm{z}=260.34$.

(S)-2-amino-N-(2-((2-amino-2-oxoethyl)amino)-2-oxoethyl)-4-methylpentanamide (LGG) ${ }^{1} \mathrm{H} \quad \mathrm{NMR}$ $\left(500 \mathrm{MHz}, \mathrm{D}_{2} \mathrm{O}, \mathrm{ppm}\right): \delta=4.13-4.06(\mathrm{~m}, 2 \mathrm{H}), 4.04-3.99(\mathrm{~m}, 1 \mathrm{H}), 3.95(\mathrm{~s}, 2 \mathrm{H}), 1.84-1.66(\mathrm{~m}, 3 \mathrm{H}), 0.98$ $(\mathrm{t}, 6 \mathrm{H}) ;{ }^{13} \mathrm{C}$ NMR $\left(125 \mathrm{MHz}, \mathrm{D}_{2} \mathrm{O}, \mathrm{ppm}\right): \delta=174.05,171.42,171.16,51.85,42.28,41.92,39.71,23.78$, 21.59, 20.96; ESI MS for $[\mathrm{M}-\mathrm{H}]^{+}:$calc. $\mathrm{m} / \mathrm{z}=245.16$, found $\mathrm{m} / \mathrm{z}=245.34$.

(S)-4-amino-5-((2-((2-amino-2-oxoethyl)amino)-2-oxoethyl)amino)-5-oxopentanoic acid (EGG) ${ }^{1} \mathrm{H}$ NMR (500 MHz, D $2 \mathrm{O}, \mathrm{ppm}): \delta=4.15(\mathrm{t}, 1 \mathrm{H}), 4.09(\mathrm{~d}, 1 \mathrm{H}), 4.02(\mathrm{~d}, 1 \mathrm{H}), 3.95(\mathrm{~s}, 2 \mathrm{H}), 2.60(\mathrm{t}, 2 \mathrm{H}), 2.21(\mathrm{q}$, $2 \mathrm{H}) ;{ }^{13} \mathrm{C} \mathrm{NMR}\left(125 \mathrm{MHz}, \mathrm{D}_{2} \mathrm{O}, \mathrm{ppm}\right): \delta=176.18,174.06,171.35,169.95,52.40,42.28,41.93,29.05,25.74$; ESI MS for $[\mathrm{M}-\mathrm{H}]^{+}:$calc. $\mathrm{m} / \mathrm{z}=261.12$, found $\mathrm{m} / \mathrm{z}=261.34$. 
(S)-2-amino-N-(14-amino-2,5,8,11,14-pentaoxo-3,6,9,12-tetraazatetradecyl)-3-(perfluorophenyl) propenamide (F'GGGGG) ${ }^{1} \mathrm{H}$ NMR $\left(700 \mathrm{MHz}, \mathrm{D}_{2} \mathrm{O}, \mathrm{ppm}\right): \delta=4.30(\mathrm{t}, 1 \mathrm{H}), 4.02(\mathrm{~m}, 8 \mathrm{H}), 3.95(\mathrm{~s}, 2 \mathrm{H})$, 3.37 (d, 2H); ${ }^{19} \mathrm{~F} \mathrm{NMR}\left(375 \mathrm{MHz}, \mathrm{D}_{2} \mathrm{O}, \mathrm{ppm}\right): \delta=-142.63,-154.54,-162.22$; ESI MS for $[\mathrm{M}-\mathrm{H}]^{+}:$calc. $\mathrm{m} / \mathrm{z}$ $=540.16$, found $\mathrm{m} / \mathrm{z}=540.67$.

2-amino-N-(14-amino-2,5,8,11,14-pentaoxo-3,6,9,12-tetraazatetradecyl)acetamide (GGGGGG) ${ }^{1} \mathrm{H}$ NMR (700 MHz, $\left.\mathrm{D}_{2} \mathrm{O}, \mathrm{ppm}\right): \delta=4.09$ (s, 2H), 4.04 (d, 4H), 4.02 (s, 2H), 3.95 (s, 2H), 3.93 (s, 2H); ESI MS for $[\mathrm{M}-\mathrm{H}]^{+}:$calc. $\mathrm{m} / \mathrm{z}=360.16$, found $\mathrm{m} / \mathrm{z}=360.50$. 


\section{SI-3 Study on heteropeptide dimerization by ${ }^{1} \mathrm{H} \&{ }^{19} \mathrm{~F}$ NMR}

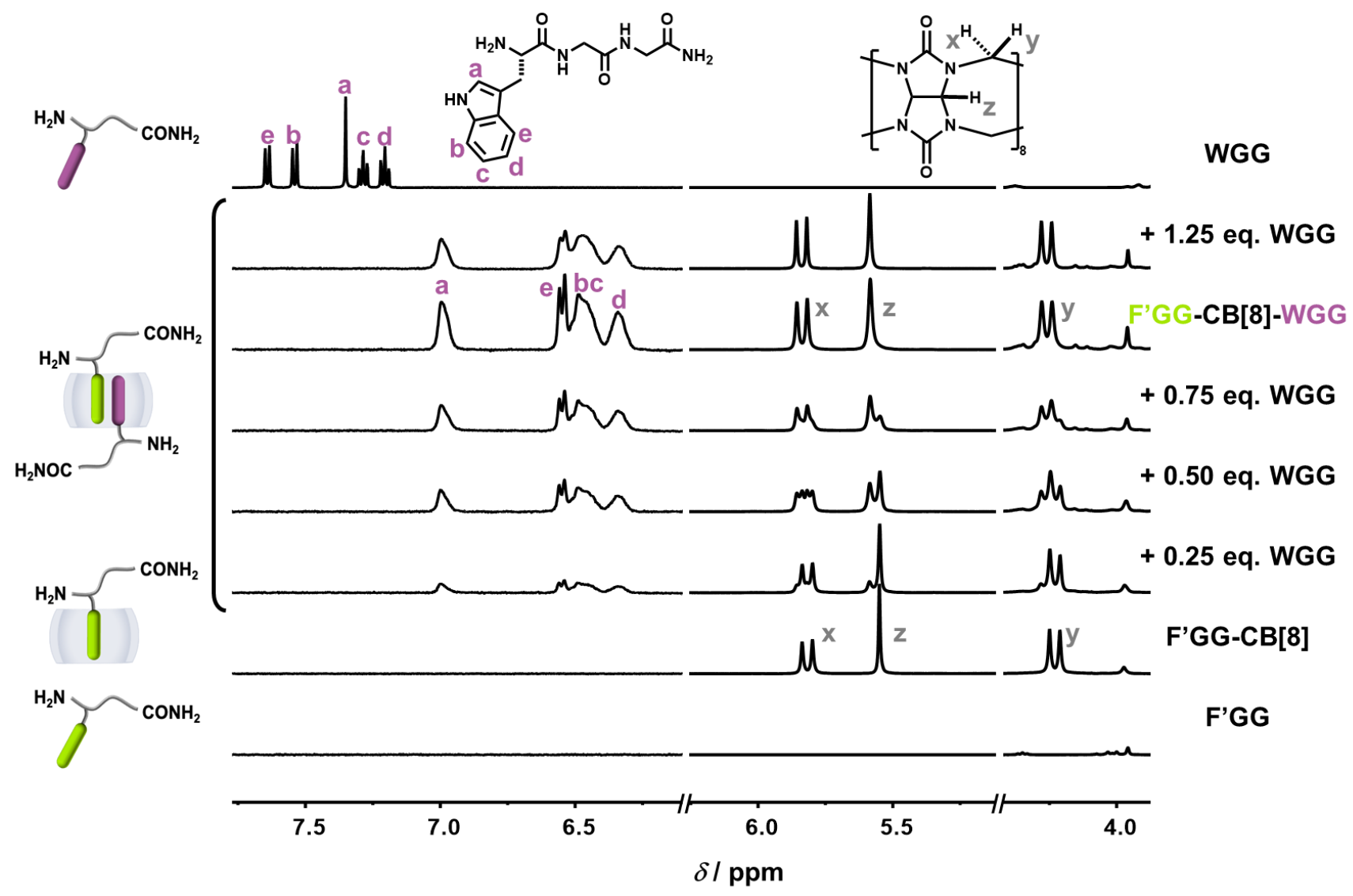

Figure S1 ${ }^{1} \mathrm{H}$ NMR titrations by adding WGG to F'GG-CB[8] (1:1 complex) at the molar ratio ranging from 0.25:1.00 to 1.25:1.00 with three controls of free F'GG, pure F'GG-CB[8] complex, free WGG. Within each area (7.6 - $6.4 \mathrm{ppm}, 5.8-5.6 \mathrm{ppm}, 4.4-3.8 \mathrm{ppm})$, the intensity of free F'GG was adjusted by a factor of $1000 \mathrm{X}$ and free WGG by $870 \mathrm{x}$. Among these areas, the intensity of all spectra in $7.6-6.4 \mathrm{ppm}$ area was adjusted by a factor of $27 \mathrm{x}\left(400 \mathrm{MHz}, 298 \mathrm{~K}, \mathrm{D}_{2} \mathrm{O}\right.$, phosphate buffer, $\left.\mathrm{pH}=7.0\right)$

As shown in Figure S1, by adding WGG to F'GG-CB[8] (1:1 complex), the aromatic proton peaks from $7.6 \mathrm{ppm}$ to $7.2 \mathrm{ppm}$ shifted to $7.0 \mathrm{ppm}$ to $6.3 \mathrm{ppm}$ and became blunt because of the encapsulation. The proton $\mathrm{x}, \mathrm{y}, \mathrm{z}$ on $\mathrm{CB}[8]$ also shifted from one state to another. This observation indicated that a new heteroternary complex of WGG-F'GG-CB[8] was formed. 


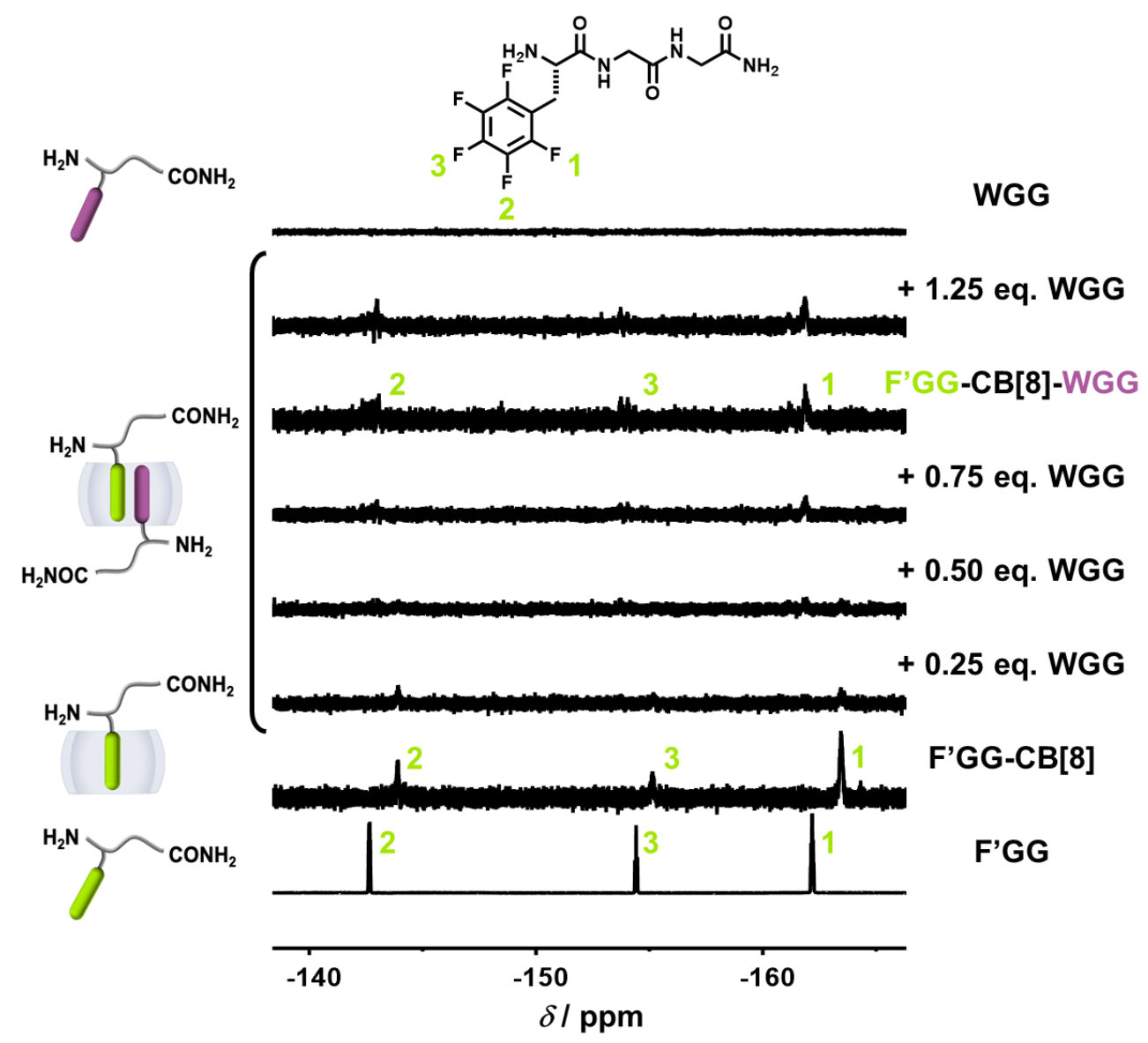

Figure S2 ${ }^{19} \mathrm{~F}$ NMR titrations by adding WGG to F'GG-CB[8] (1:1 complex) at the molar ratio ranging from 0.25:1.00 to 1.25:1.00 with three controls of free F' GG, pure F' GG-CB[8] complex, free WGG. The intensity of free F'GG was adjusted by a factor 0.01 x (400 MHz, $298 \mathrm{~K}, \mathrm{D}_{2} \mathrm{O}$, phosphate buffer, $\left.\mathrm{pH}=7.0\right)$

As shown in Figure S2, by adding WGG to F'GG-CB[8] (1:1 complex), the peaks of fluorine atom 1, 2, 3 at $-162.2,-142.6,-154.4 \mathrm{ppm}$ shifted downfield and then gradually moved upfield along with the addition of WGG. This observation is consistent with Figure S1 and indicated a new heteroternary complex of WGGF'GG-CB[8] was formed. 


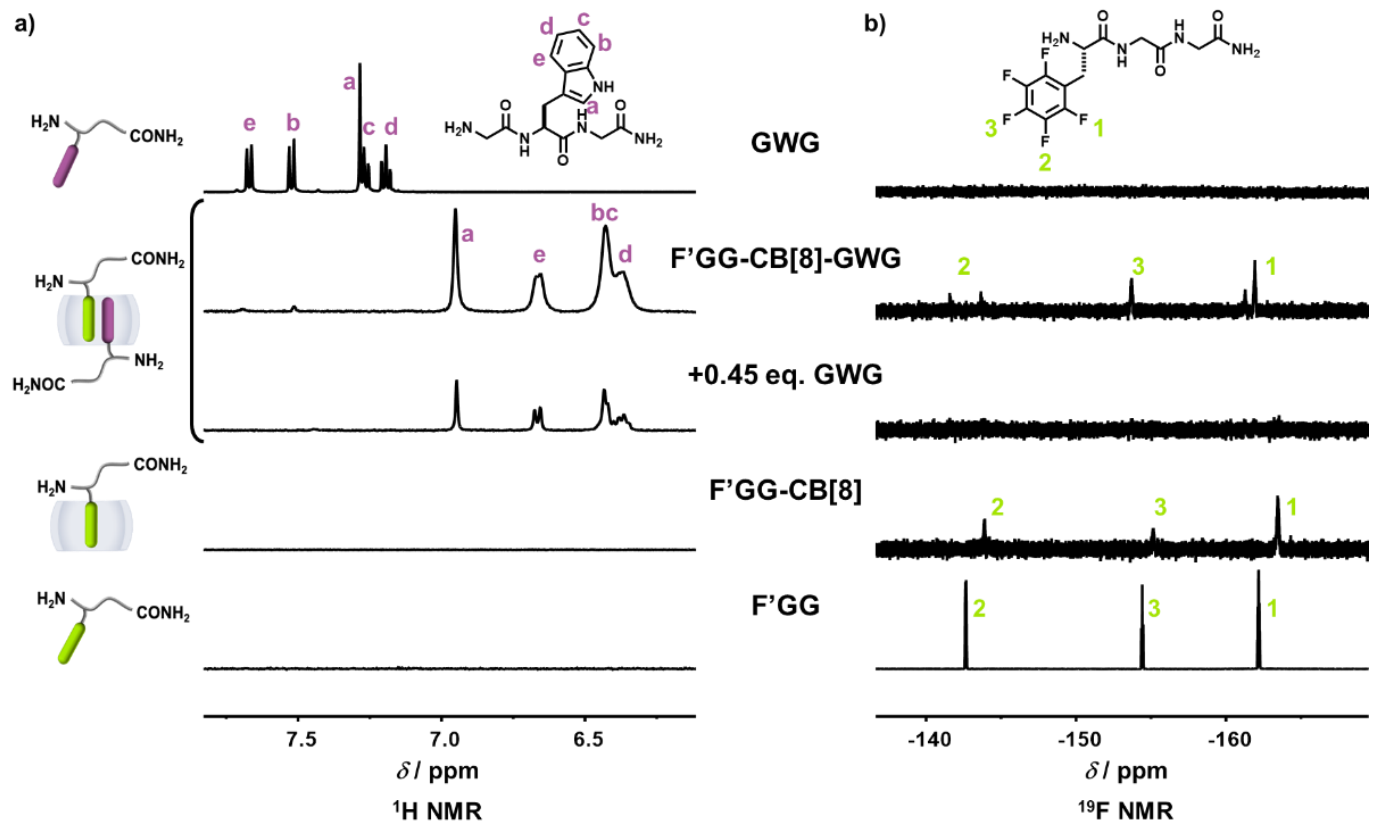

Figure S3 a) ${ }^{1} \mathrm{H}$ and b) ${ }^{19} \mathrm{~F}$ NMR titrations by adding GWG to F'GG-CB[8] (1:1 complex) at the molar ratio 0.45:1.00 and 1.00:1.00 with three controls of free F'GG, pure F'GG-CB[8] complex and free GWG. The ${ }^{1} \mathrm{H}$ NMR spectrum intensity of free GWG was adjusted by a factor of $100 \mathrm{x}$ and ${ }^{19} \mathrm{~F}$ NMR spectrum intensity of free F'GG by 0.0002 x. (400 MHz, $298 \mathrm{~K}, \mathrm{D}_{2} \mathrm{O}$, phosphate buffer, $\mathrm{pH}=7.0$ )

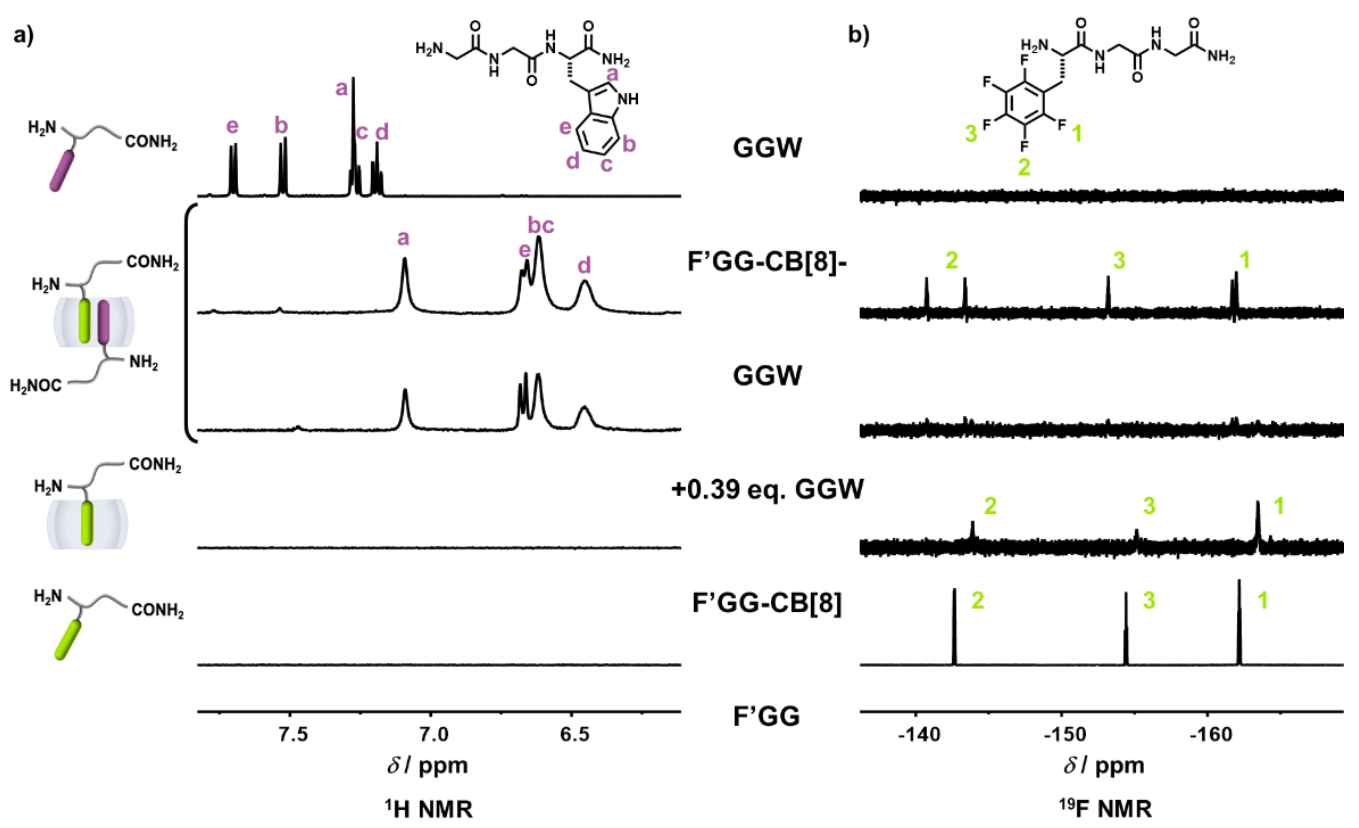

Figure S4 a) ${ }^{1} \mathrm{H}$ and b) ${ }^{19} \mathrm{~F}$ NMR titrations by adding GGW to F'GG-CB[8] (1:1 complex) at the molar ratio 0.39:1.00 and 1.00:1.00 with three controls of free F'GG, pure F'GG-CB[8] complex and free GGW. The ${ }^{1} \mathrm{H}$ NMR spectrum intensity of free GGW was adjusted by a factor of $73 \mathrm{x}$ and ${ }^{19} \mathrm{~F}$ NMR spectrum intensity of free F'GG by 0.0002 x. (400 MHz, $298 \mathrm{~K}, \mathrm{D}_{2} \mathrm{O}$, phosphate buffer, $\left.\mathrm{pH}=7.0\right)$ 
a)

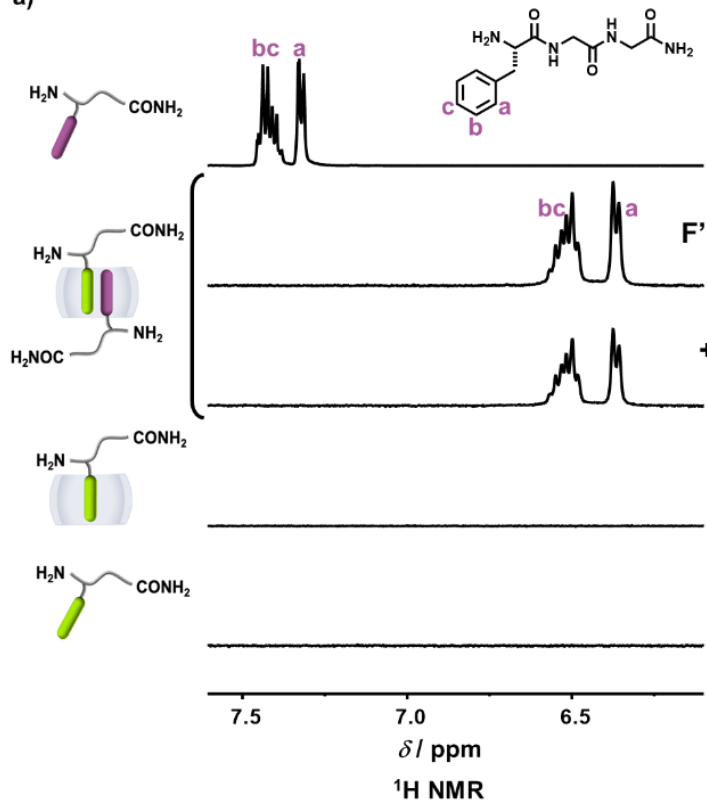

b)

FGG

F'GG-CB[8]-FGG

+0.50 eq. FGG

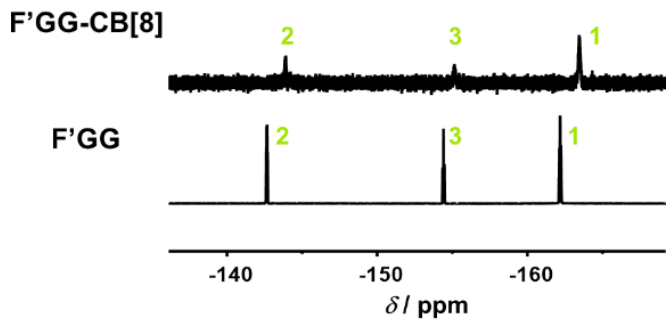

${ }^{19} \mathrm{~F}$ NMR

Figure S5 a) ${ }^{1} \mathrm{H}$ and b) ${ }^{19} \mathrm{~F}$ NMR titrations by adding FGG to F'GG-CB[8] (1:1 complex) at the molar ratio 0.50:1.00 and 1.00:1.00 with three controls of free F'GG, pure F'GG-CB[8] complex and free FGG. The ${ }^{1} \mathrm{H}$ NMR spectrum intensity of free FGG was adjusted by a factor of $80 \mathrm{x}$ and ${ }^{19} \mathrm{~F}$ NMR spectrum intensity of free F'GG by 0.0002 x. (400 MHz, $298 \mathrm{~K}, \mathrm{D}_{2} \mathrm{O}$, phosphate buffer, $\mathrm{pH}=7.0$ )

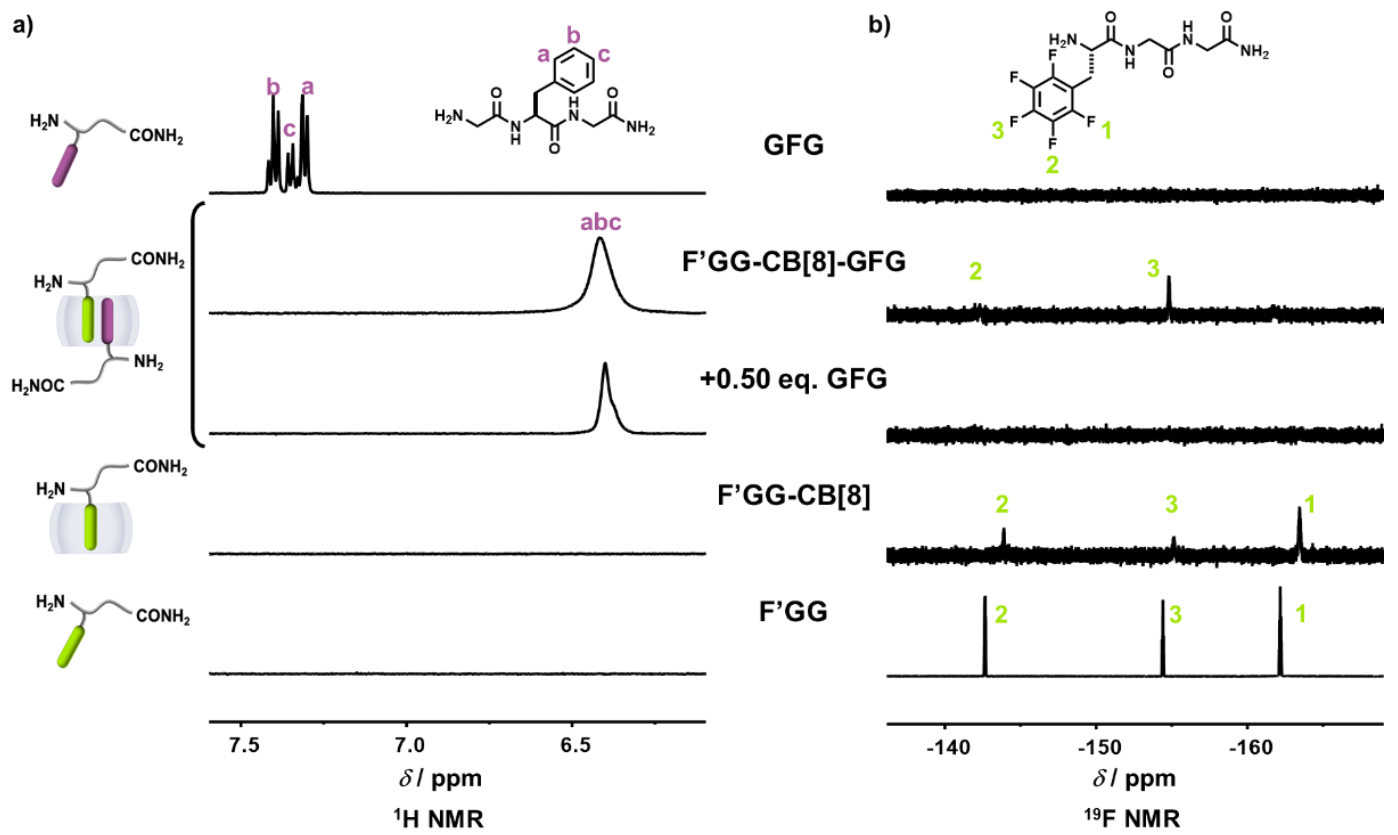

Figure S6 a) ${ }^{1} \mathrm{H}$ and b) ${ }^{19} \mathrm{~F}$ NMR titrations by adding GFG to F'GG-CB[8] (1:1 complex) at the molar ratio 0.50:1.00 and 1.00:1.00 with three controls of free F'GG, pure F'GG-CB[8] complex and free GFG. The ${ }^{1} \mathrm{H}$ NMR spectrum intensity of free GFG was adjusted by a factor of $22 \mathrm{x}$ and ${ }^{19} \mathrm{~F}$ NMR spectrum intensity of free F'GG by 0.0002 x. (400 MHz, $298 \mathrm{~K}, \mathrm{D}_{2} \mathrm{O}$, phosphate buffer, $\mathrm{pH}=7.0$ ) 
a)

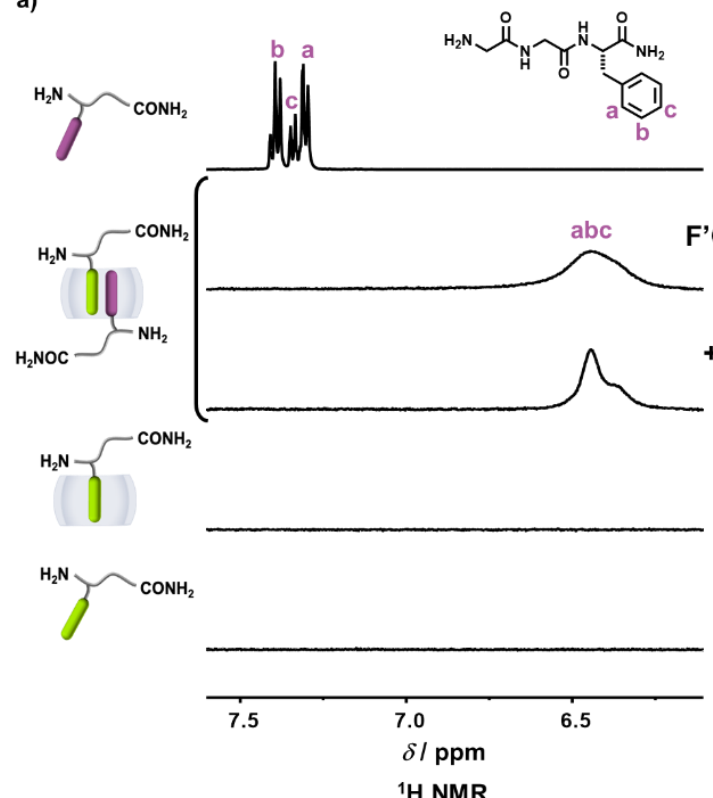

b)

GGF

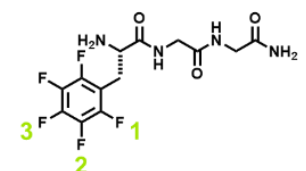

'GG-CB[8]-GGF

+0.50 eq. GGF

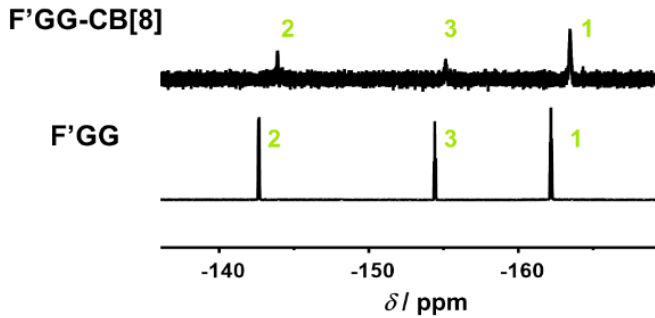

${ }^{19} \mathrm{~F}$ NMR

Figure S7 a) ${ }^{1} \mathrm{H}$ and b) ${ }^{19} \mathrm{~F}$ NMR titrations by adding GGF to F' GG-CB[8] (1:1 complex) at the molar ratio 0.50:1.00 and 1.00:1.00 with three controls of free F'GG, pure F'GG-CB[8] complex and free GGF. The ${ }^{1} \mathrm{H}$ NMR spectrum intensity of free GGF was adjusted by a factor of $9 \mathrm{x}$ and ${ }^{19} \mathrm{~F}$ NMR spectrum intensity of free F'GG by 0.0002 x. (400 MHz, $298 \mathrm{~K}, \mathrm{D}_{2} \mathrm{O}$, phosphate buffer, $\mathrm{pH}=7.0$ )

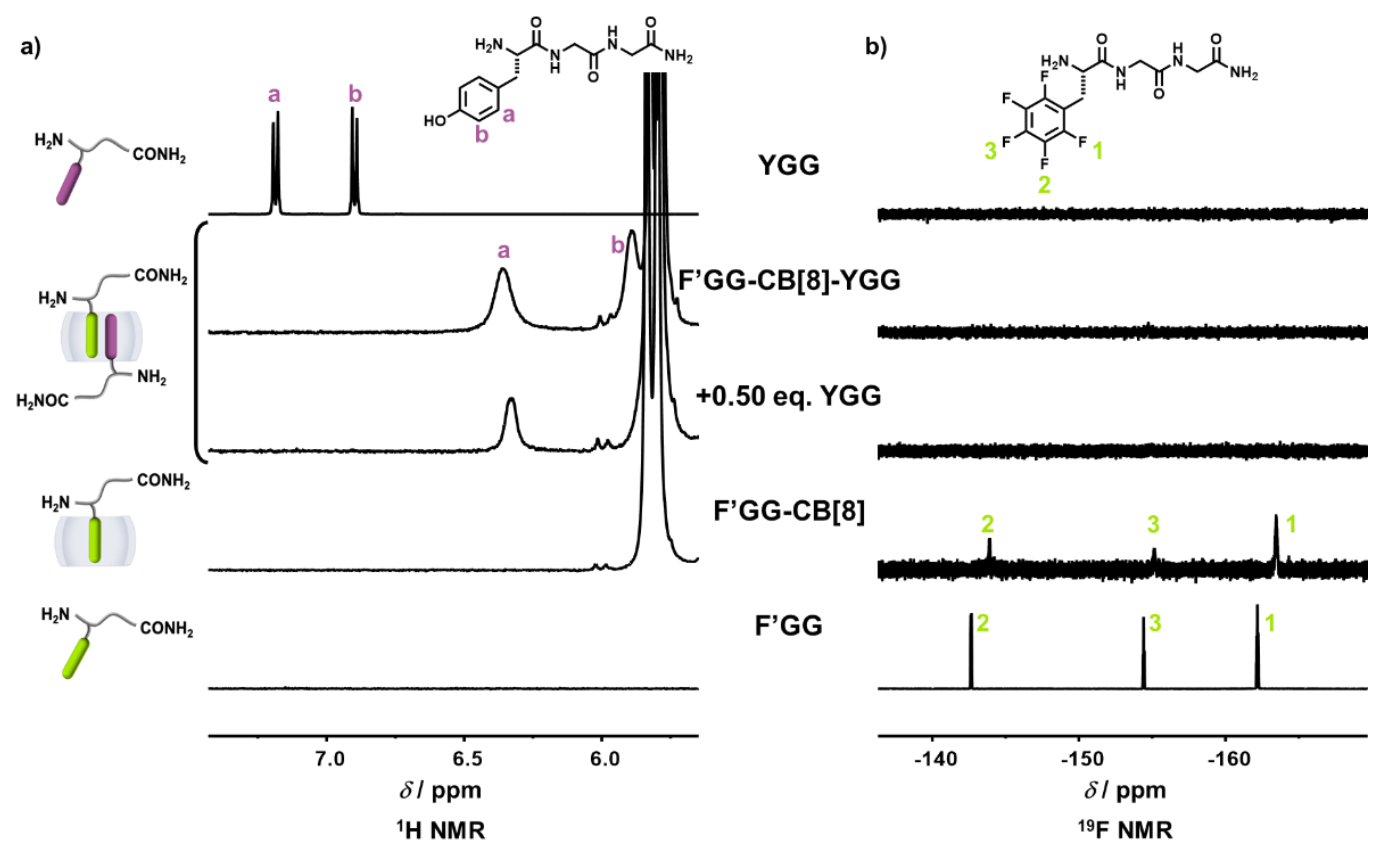

Figure S8 a) ${ }^{1} \mathrm{H}$ and b) ${ }^{19} \mathrm{~F}$ NMR titrations by adding YGG to F' GG-CB[8] (1:1 complex) at the molar ratio 0.50:1.00 and 1.00:1.00 with three controls of free F'GG, pure F'GG-CB[8] complex and free YGG. The ${ }^{1} \mathrm{H}$ NMR spectrum intensity of free YGG was adjusted by a factor of $20 \mathrm{x}$ and ${ }^{19} \mathrm{~F}$ NMR spectrum intensity of free F'GG by 0.0002 x. (400 MHz, $298 \mathrm{~K}, \mathrm{D}_{2} \mathrm{O}$, phosphate buffer, $\mathrm{pH}=7.0$ ) 


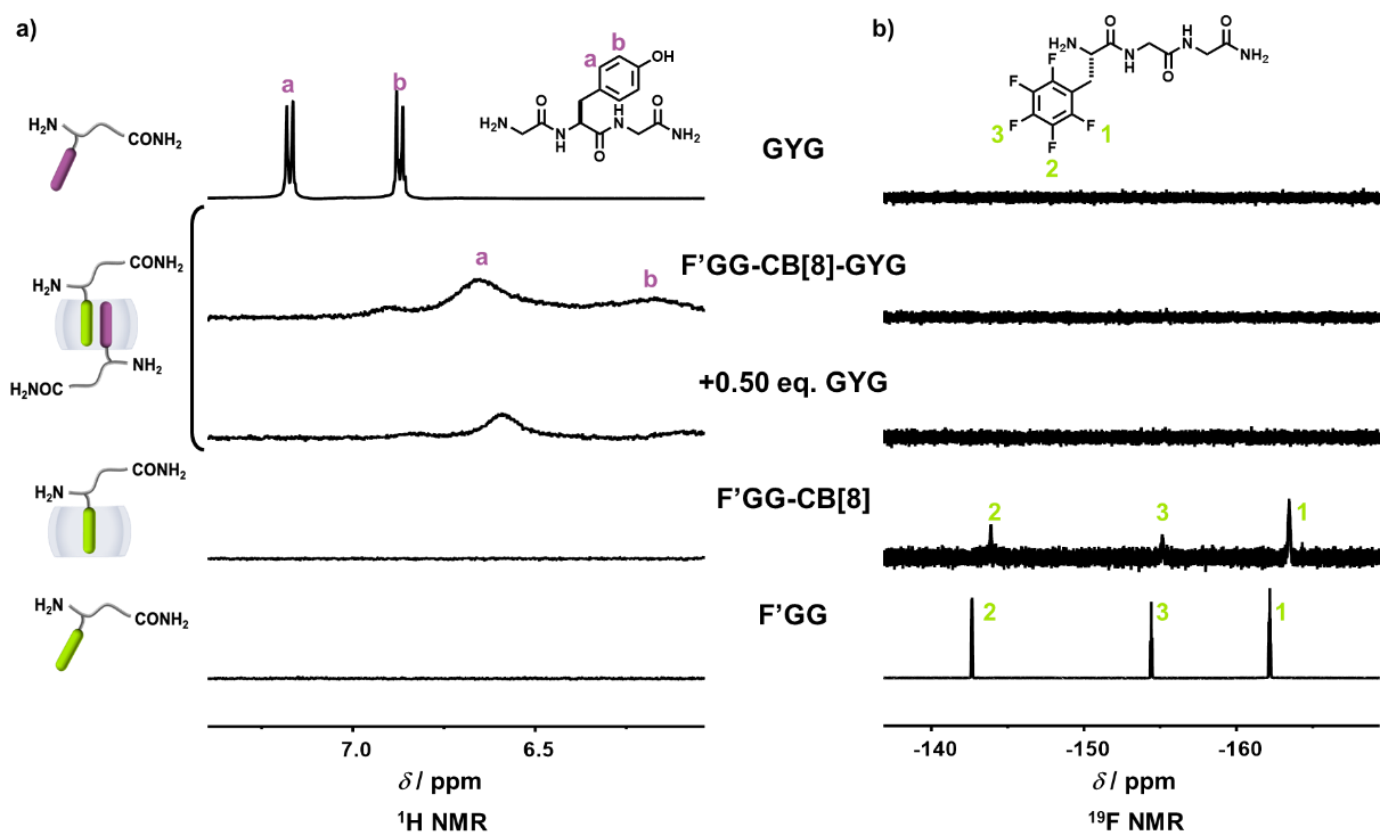

Figure S9 a) ${ }^{1} \mathrm{H}$ and b) ${ }^{19} \mathrm{~F}$ NMR titrations by adding GYG to F' GG-CB[8] (1:1 complex) at the molar ratio 0.50:1.00 and 1.00:1.00 with three controls of free F'GG, pure F'GG-CB[8] complex and free GYG. The ${ }^{1} \mathrm{H}$ NMR spectrum intensity of free GYG was adjusted by a factor of $6 \mathrm{x}$ and ${ }^{19} \mathrm{~F}$ NMR spectrum intensity of free F'GG by 0.0002 x. (400 MHz, $298 \mathrm{~K}, \mathrm{D}_{2} \mathrm{O}$, phosphate buffer, $\mathrm{pH}=7.0$ )

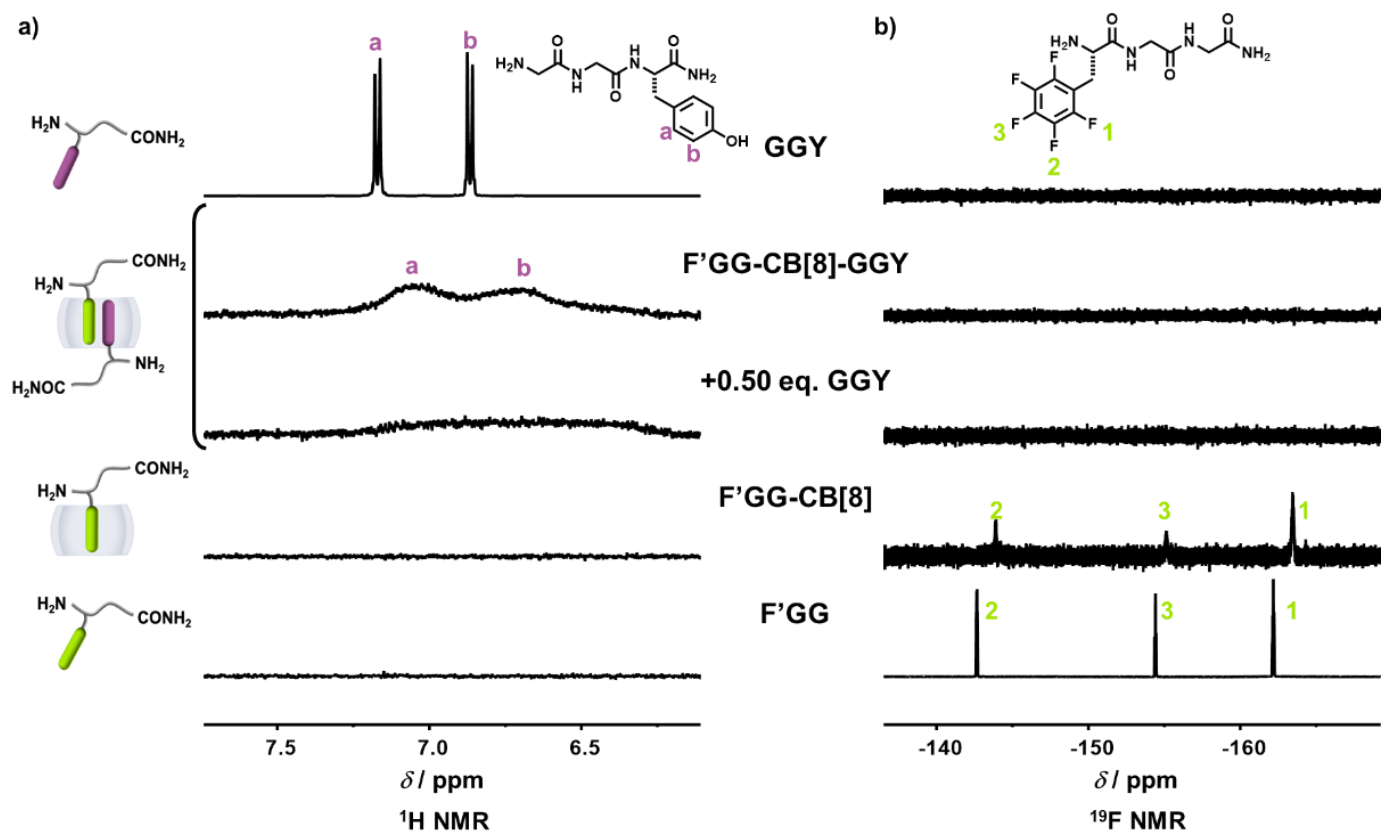

Figure S10 a ${ }^{1} \mathrm{H}$ and b) ${ }^{19} \mathrm{~F}$ NMR titrations by adding GGY to F'GG-CB[8] (1:1 complex) at the molar ratio 0.50:1.00 and 1.00:1.00 with three controls of free F'GG, pure F'GG-CB[8] complex and free GGY. The ${ }^{1} \mathrm{H}$ NMR spectrum intensity of free GGY was adjusted by a factor of $6 \mathrm{x}$ and ${ }^{19} \mathrm{~F}$ NMR spectrum intensity of free F'GG by 0.0002 x. (400 MHz, $298 \mathrm{~K}, \mathrm{D}_{2} \mathrm{O}$, phosphate buffer, $\mathrm{pH}=7.0$ ) 


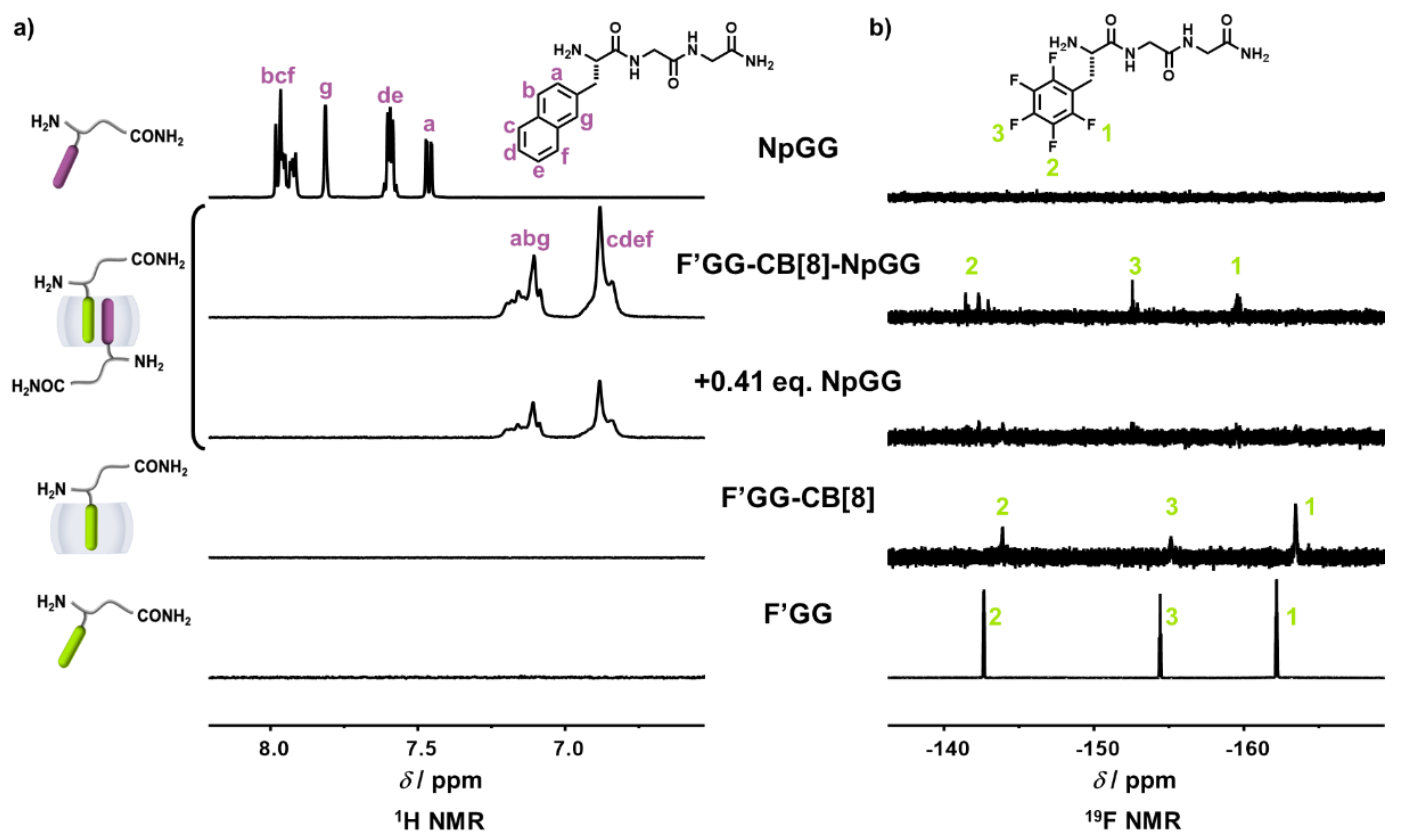

Figure S11 a) ${ }^{1} \mathrm{H}$ and b) ${ }^{19} \mathrm{~F}$ NMR titrations by adding NpGG to F'GG-CB[8] (1:1 complex) at the molar ratio 0.41:1.00 and 1.00:1.00 with three controls of free F'GG, pure F'GG-CB[8] complex and free NpGG. The ${ }^{1} \mathrm{H}$ NMR spectrum intensity of free NpGG was adjusted by a factor of $61 \mathrm{x}$ and ${ }^{19} \mathrm{~F}$ NMR spectrum intensity of free F'GG by 0.0002 x. (400 MHz, $298 \mathrm{~K}, \mathrm{D}_{2} \mathrm{O}$, phosphate buffer, $\mathrm{pH}=7.0$ )

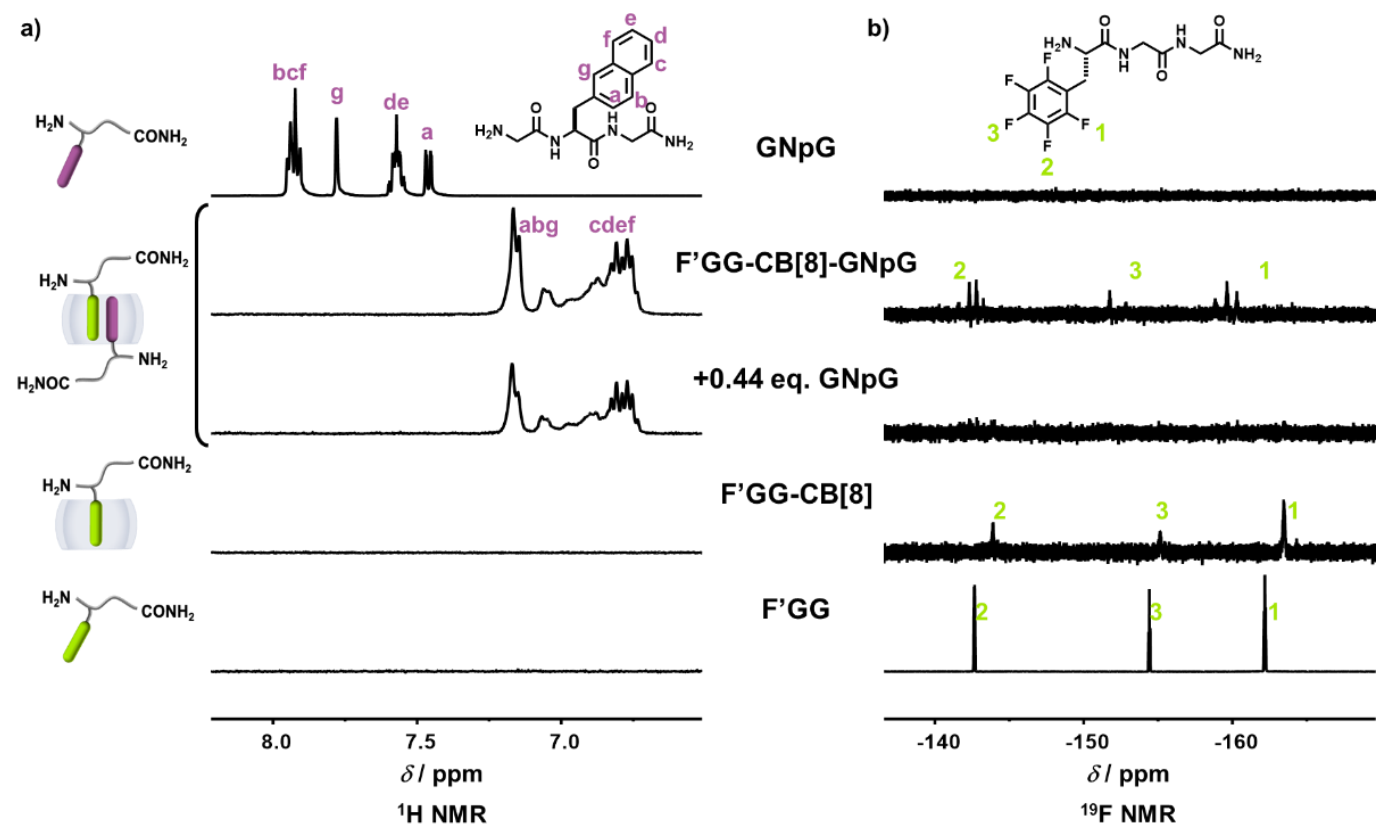

Figure S12 a) ${ }^{1} \mathrm{H}$ and b) ${ }^{19} \mathrm{~F}$ NMR titrations by adding GNpG to F'GG-CB[8] (1:1 complex) at the molar ratio 0.44:1.00 and 1.00:1.00 with three controls of free F'GG, pure F'GG-CB[8] complex and free GNpG. The ${ }^{1} \mathrm{H}$ NMR spectrum intensity of free GNpG was adjusted by a factor of $80 \mathrm{x}$ and ${ }^{19} \mathrm{~F}$ NMR spectrum intensity of free F'GG by 0.0002 x. (400 MHz, $298 \mathrm{~K}, \mathrm{D}_{2} \mathrm{O}$, phosphate buffer, $\mathrm{pH}=7.0$ ) 


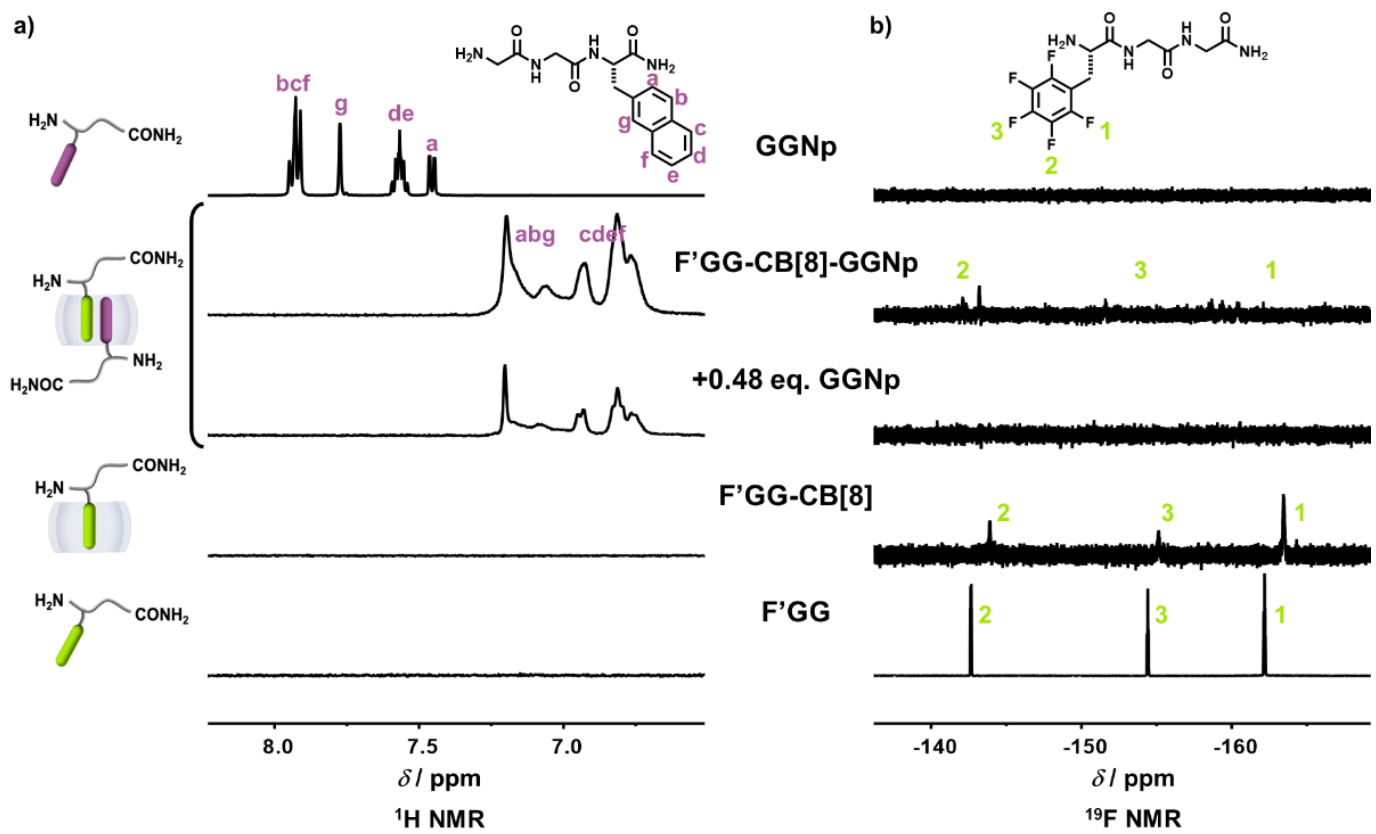

Figure S13 a) ${ }^{1} \mathrm{H}$ and b) ${ }^{19} \mathrm{~F}$ NMR titrations by adding GGNp to F'GG-CB[8] (1:1 complex) at the molar ratio 0.48:1.00 and 1.00:1.00 with three controls of free F'GG, pure F'GG-CB[8] complex and free GGNp. The ${ }^{1} \mathrm{H}$ NMR spectrum intensity of free GGNp was adjusted by a factor of $63 \mathrm{x}$ and ${ }^{19} \mathrm{~F}$ NMR spectrum intensity of free F'GG by 0.0002 x. (400 MHz, $298 \mathrm{~K}, \mathrm{D}_{2} \mathrm{O}$, phosphate buffer, $\mathrm{pH}=7.0$ )

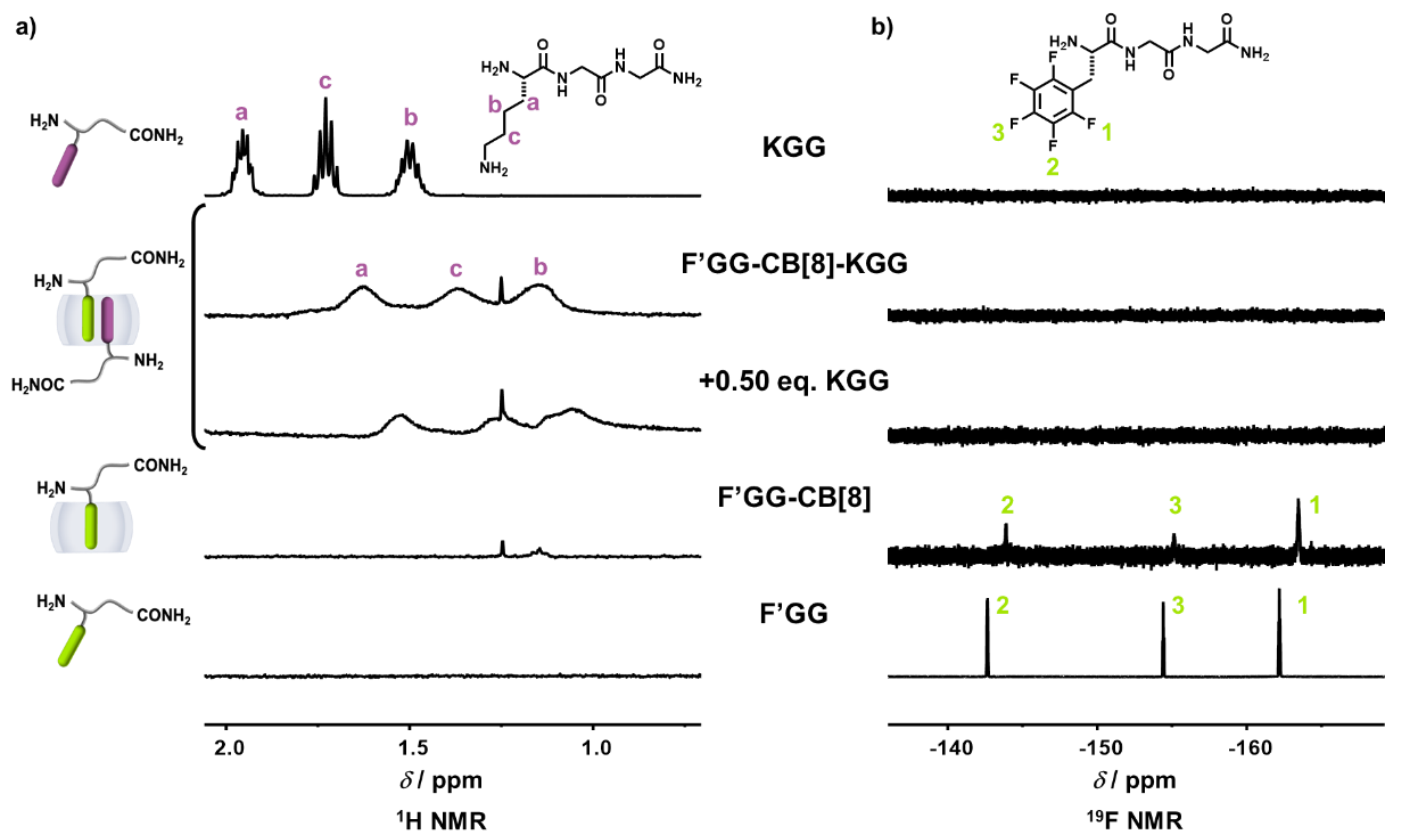

Figure S14 a) ${ }^{1} \mathrm{H}$ and b) ${ }^{19} \mathrm{~F}$ NMR titrations by adding KGG to F'GG-CB[8] (1:1 complex) at the molar ratio 0.50:1.00 and 1.00:1.00 with three controls of free F'GG, pure F'GG-CB[8] complex and free KGG. The ${ }^{1} \mathrm{H}$ NMR spectrum intensity of free KGG was adjusted by a factor of $17 \mathrm{x}$ and ${ }^{19} \mathrm{~F}$ NMR spectrum intensity of free F'GG by 0.0002 x. (400 MHz, $298 \mathrm{~K}, \mathrm{D}_{2} \mathrm{O}$, phosphate buffer, $\mathrm{pH}=7.0$ ) 


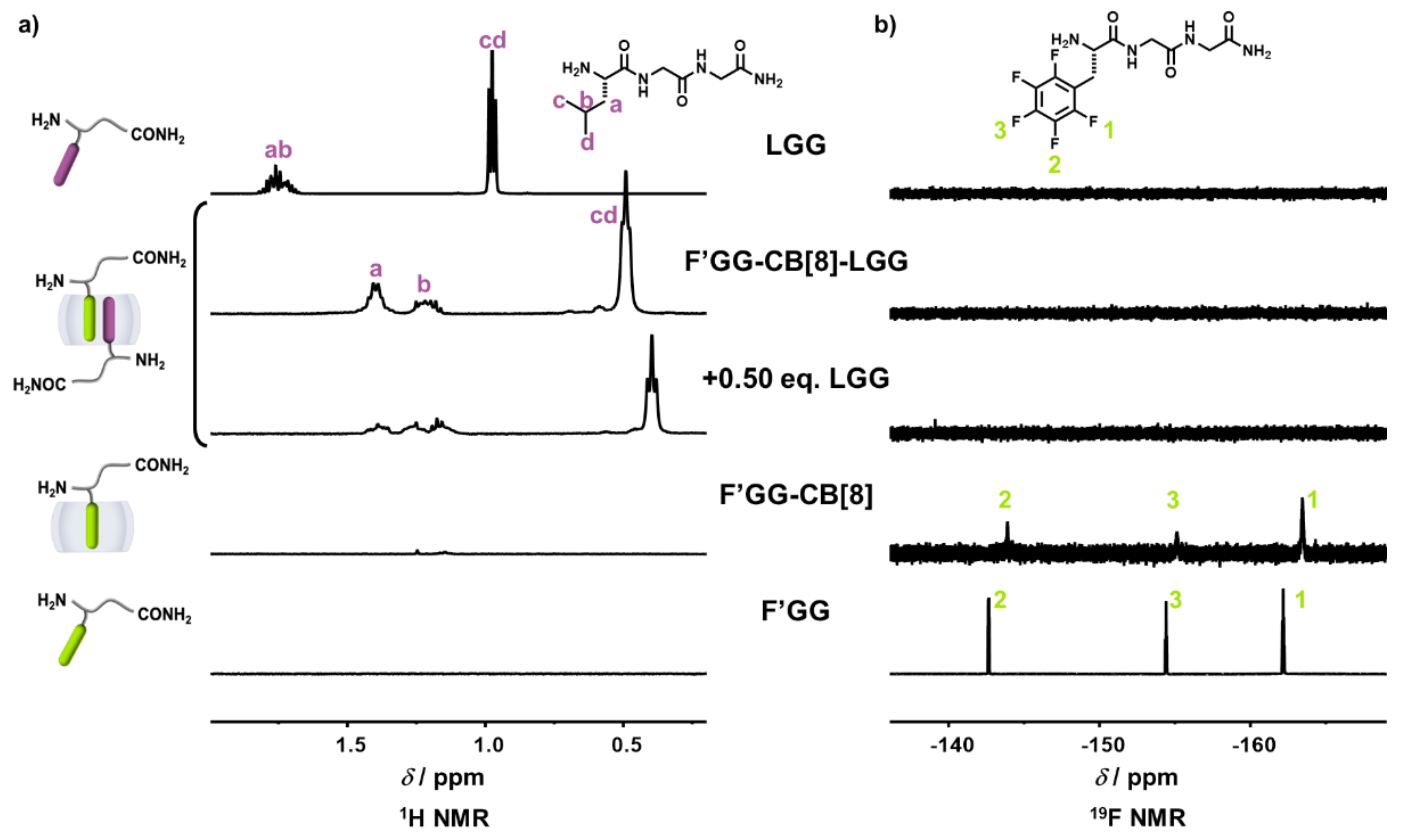

Figure S15 a) ${ }^{1} \mathrm{H}$ and b) ${ }^{19} \mathrm{~F}$ NMR titrations by adding LGG to F'GG-CB[8] (1:1 complex) at the molar ratio 0.50:1.00 and 1.00:1.00 with three controls of free F'GG, pure F'GG-CB[8] complex and free LGG. The ${ }^{1} \mathrm{H}$ NMR spectrum intensity of free LGG was adjusted by a factor of $24 \mathrm{x}$ and ${ }^{19} \mathrm{~F}$ NMR spectrum intensity of free F'GG by 0.0002 x. (400 MHz, $298 \mathrm{~K}, \mathrm{D}_{2} \mathrm{O}$, phosphate buffer, $\mathrm{pH}=7.0$ )

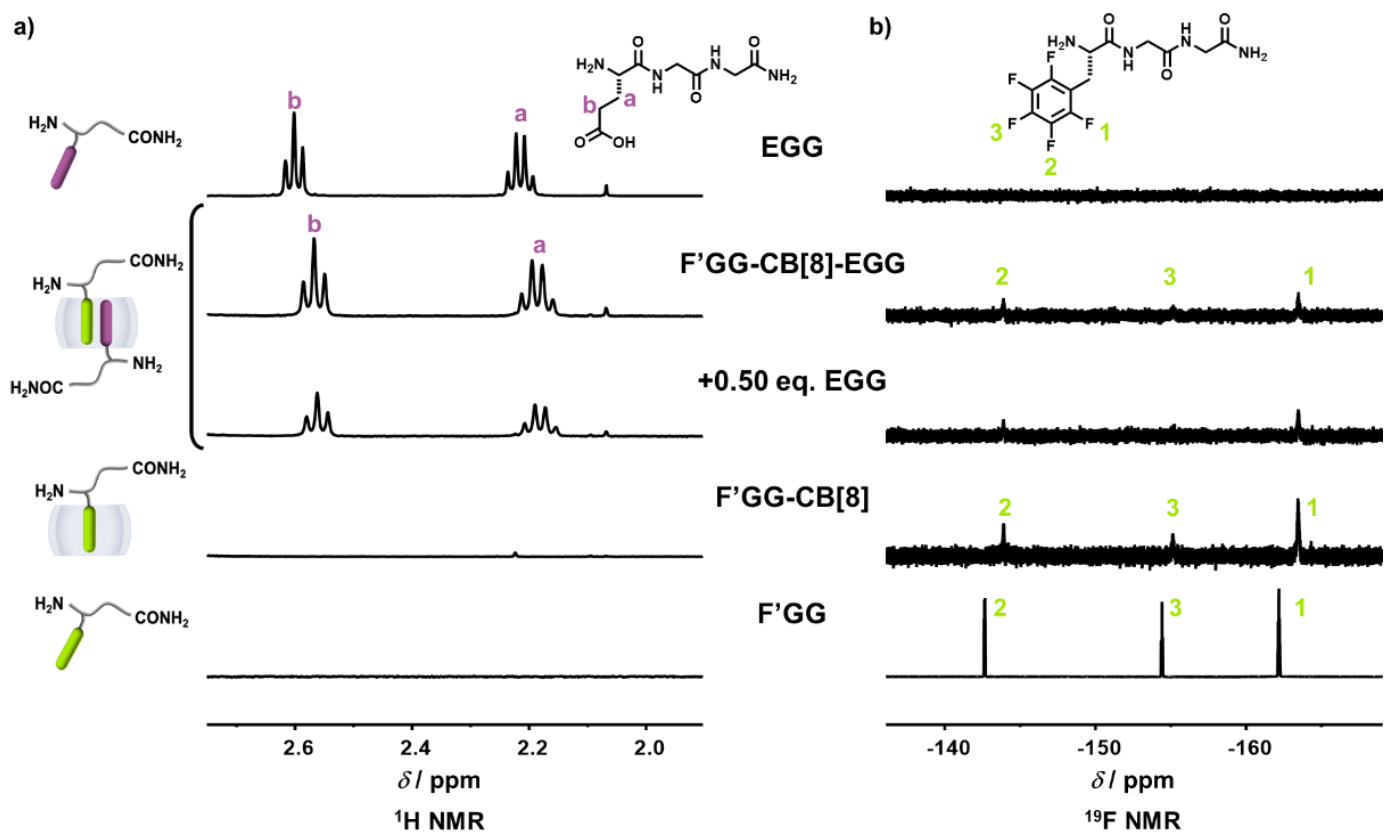

Figure S16 a) ${ }^{1} \mathrm{H}$ and b) ${ }^{19} \mathrm{~F}$ NMR titrations by adding EGG to F'GG-CB[8] (1:1 complex) at the molar ratio 0.50:1.00 and 1.00:1.00 with three controls of free F'GG, pure F'GG-CB[8] complex and free EGG. The ${ }^{1} \mathrm{H}$ NMR spectrum intensity of free EGG was adjusted by a factor of $25 \mathrm{x}$ and ${ }^{19} \mathrm{~F}$ NMR spectrum intensity of free F'GG by 0.0002 x. (400 MHz, $298 \mathrm{~K}, \mathrm{D}_{2} \mathrm{O}$, phosphate buffer, $\mathrm{pH}=7.0$ ) 


\section{SI-4 Thermodynamic investigations by ITC}

a)

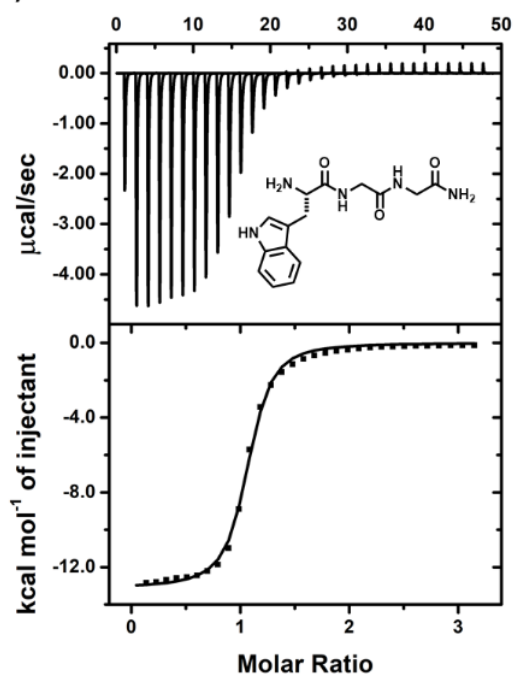

b)

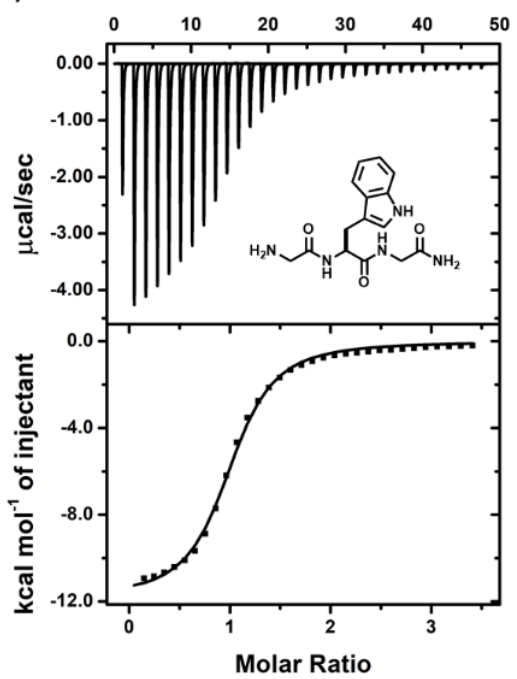

c)

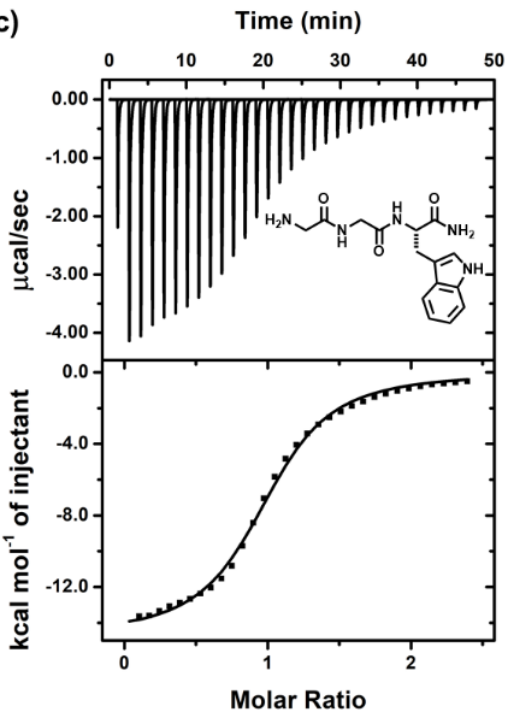

Figure S17 Representative ITC titration curves obtained by titrating a) WGG; b) GWG; c) GGW into F'GG$\mathrm{CB}[8]$ (1:1 complex), respectively. All ITC experiments were repeated for 3 times. $(298 \mathrm{~K}, 10 \mathrm{mM}$ phosphate buffer, $\mathrm{pH}=7.0$ )

a)

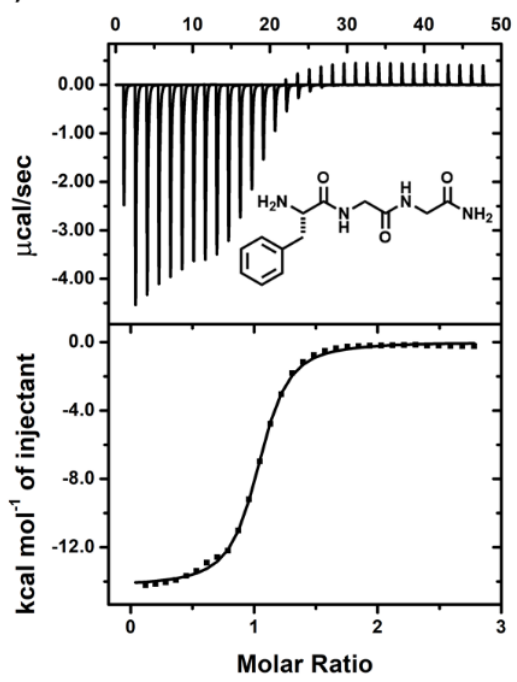

b)

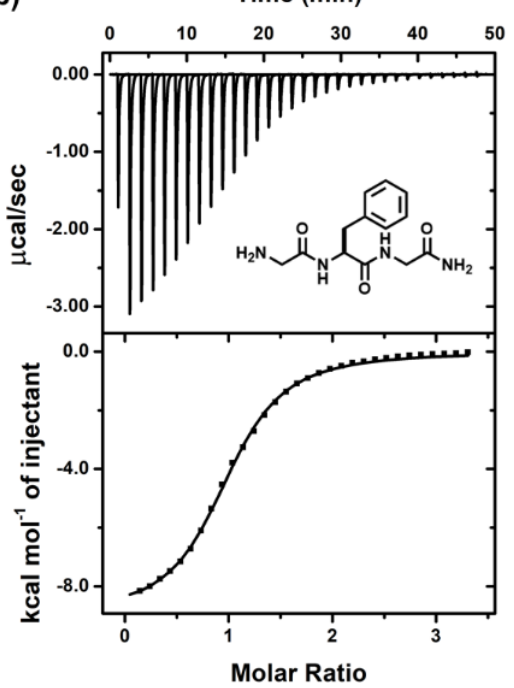

c)

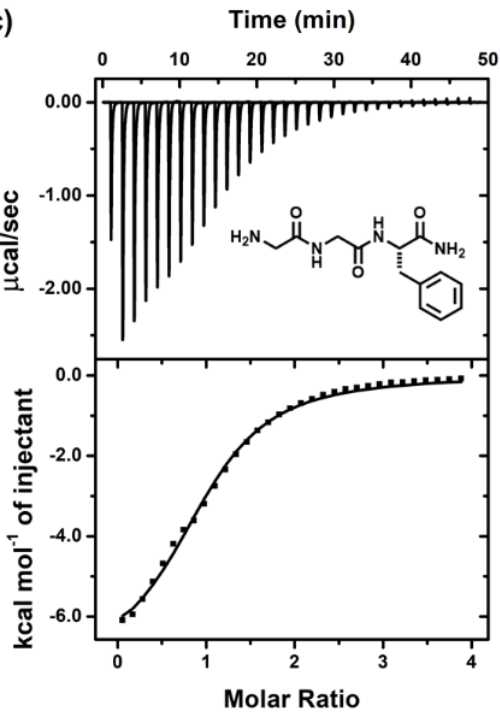

Figure S18 Representative ITC titration curves obtained by titrating a) FGG; b) GFG; c) GGF into F'GG$\mathrm{CB}[8]$ (1:1 complex), respectively. All ITC experiments were repeated for 3 times. $(298 \mathrm{~K}, 10 \mathrm{mM}$ phosphate buffer, $\mathrm{pH}=7.0$ ) 

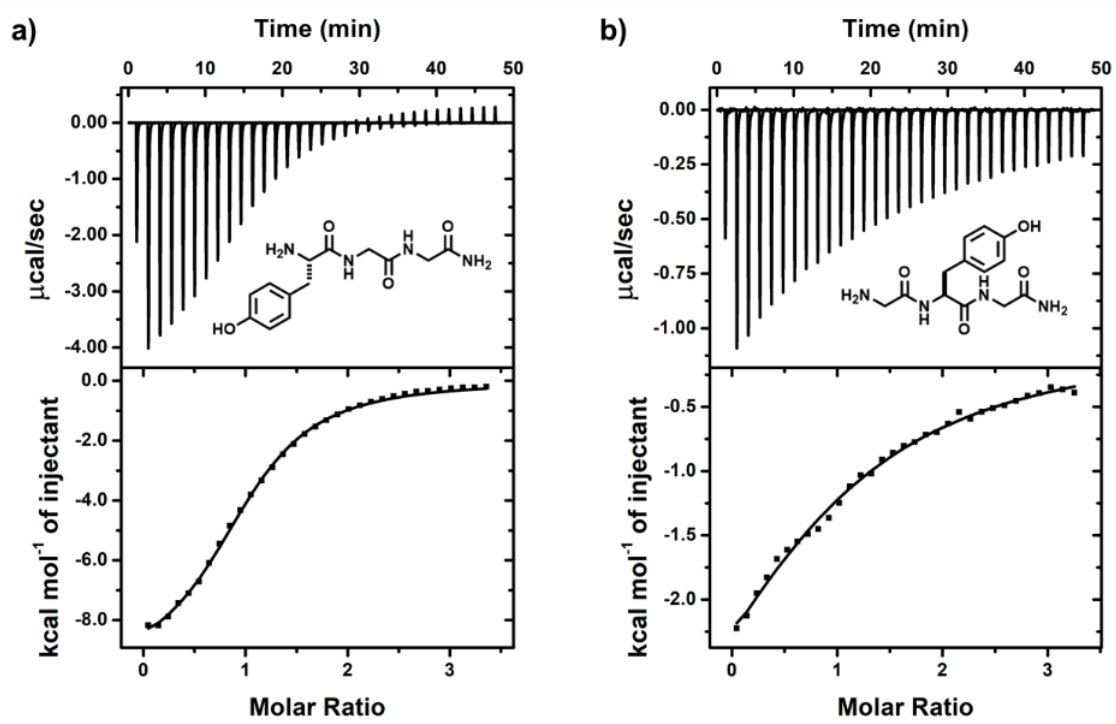

Figure S19 Representative ITC titration curves obtained by titrating a) YGG; b) GYG into F'GG-CB[8] (1:1 complex), respectively. All ITC experiments were repeated for 3 times. (298 K, $10 \mathrm{mM}$ phosphate buffer, $\mathrm{pH}=7.0)$
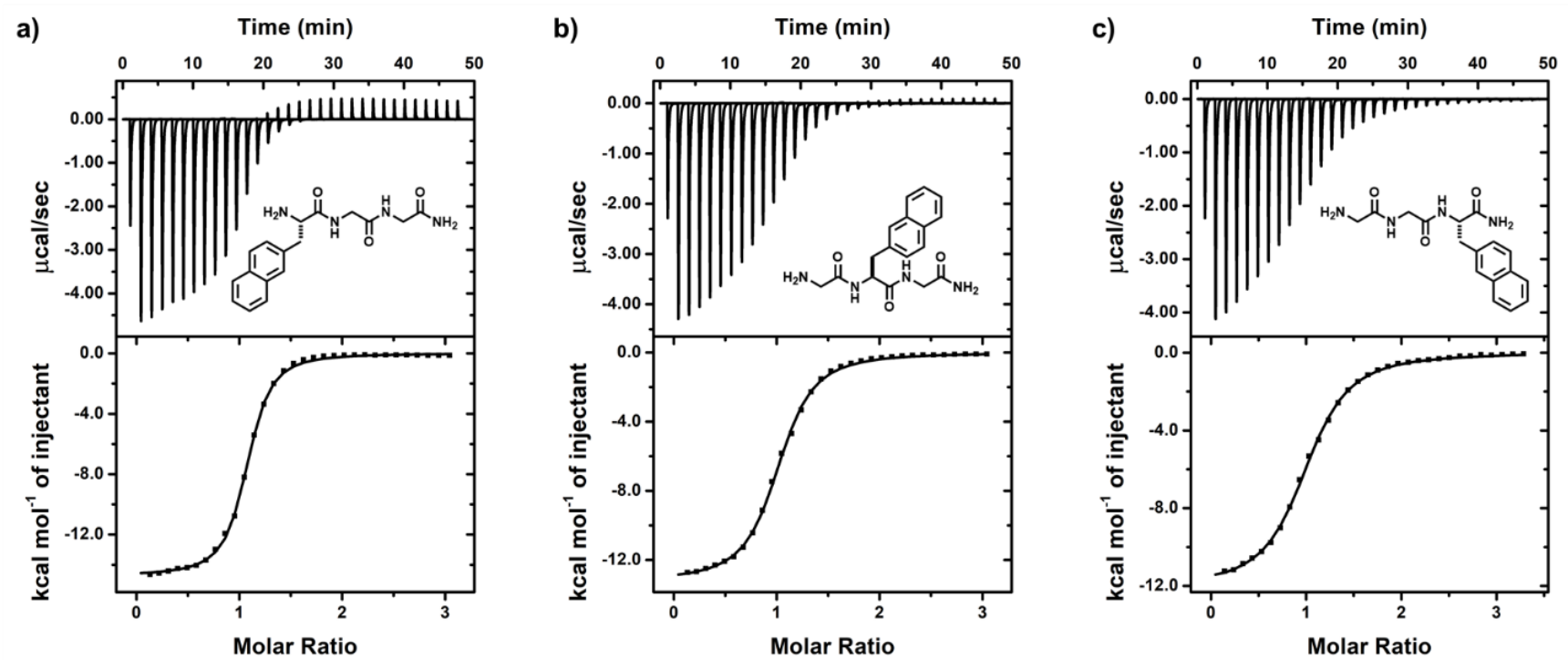

Figure S20 Representative ITC titration curves obtained by titrating a) NpGG; b) GNpG; c) GGNp into F'GG-CB[8] (1:1 complex), respectively. All ITC experiments were repeated for 3 times. (298 K, $10 \mathrm{mM}$ phosphate buffer, $\mathrm{pH}=7.0$ )

The complexation of GGY, KGG, LGG, EGG with F'GG-CB[8] were too weak $\left(K_{\mathrm{a}}<10^{2} \mathrm{M}^{-1}\right)$ to be determined by ITC so that their non-binding curve (close to baseline) was not shown here. 

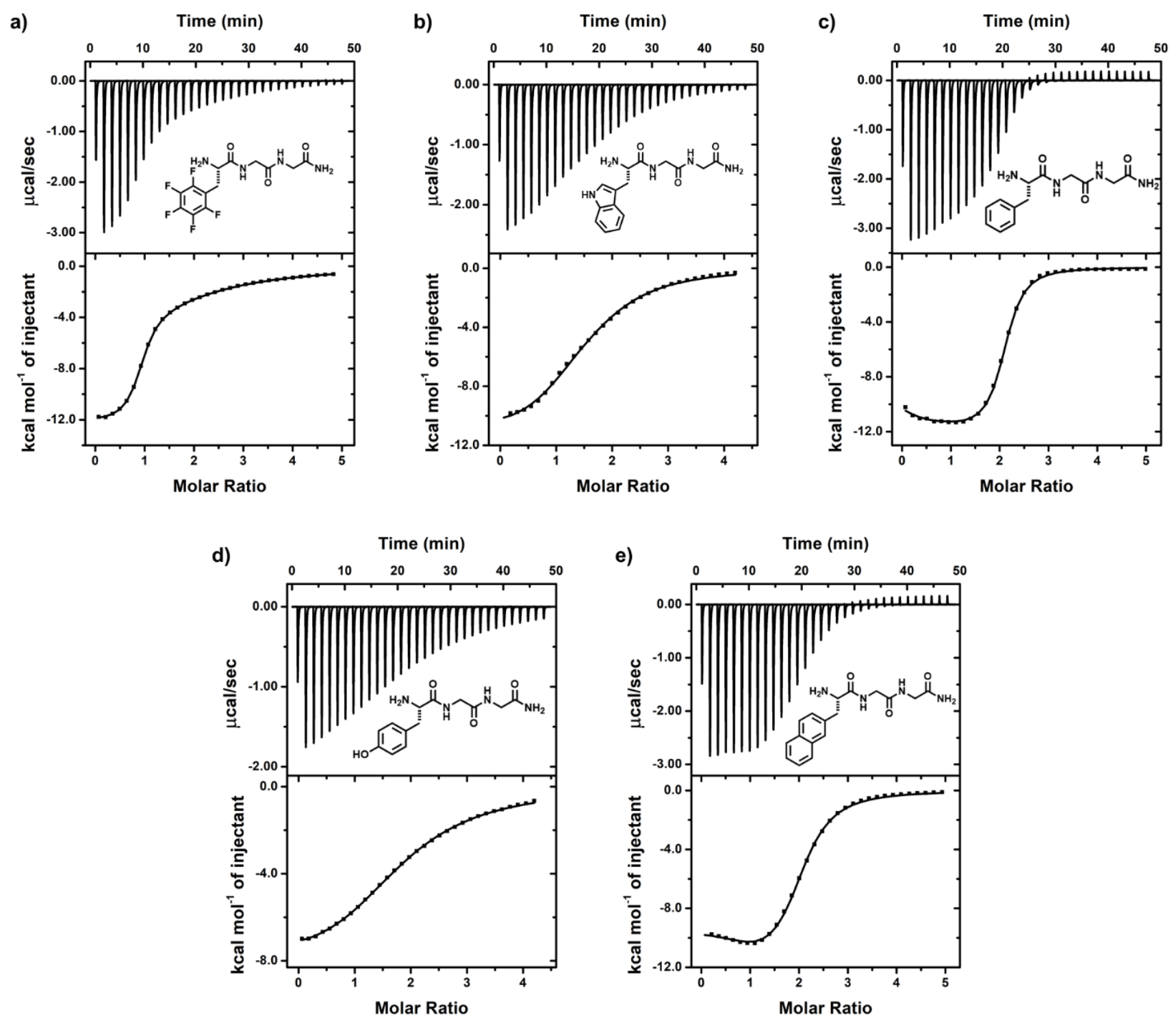

Figure S21 Representative ITC titration curves obtained by titrating a) F'GG; b) WGG; c) FGG; d) YGG; e) NpGG into CB[8], respectively. All ITC experiments were repeated for 3 times. $(298 \mathrm{~K}, 10 \mathrm{mM}$ phosphate buffer, $\mathrm{pH}=7.0$ ) 

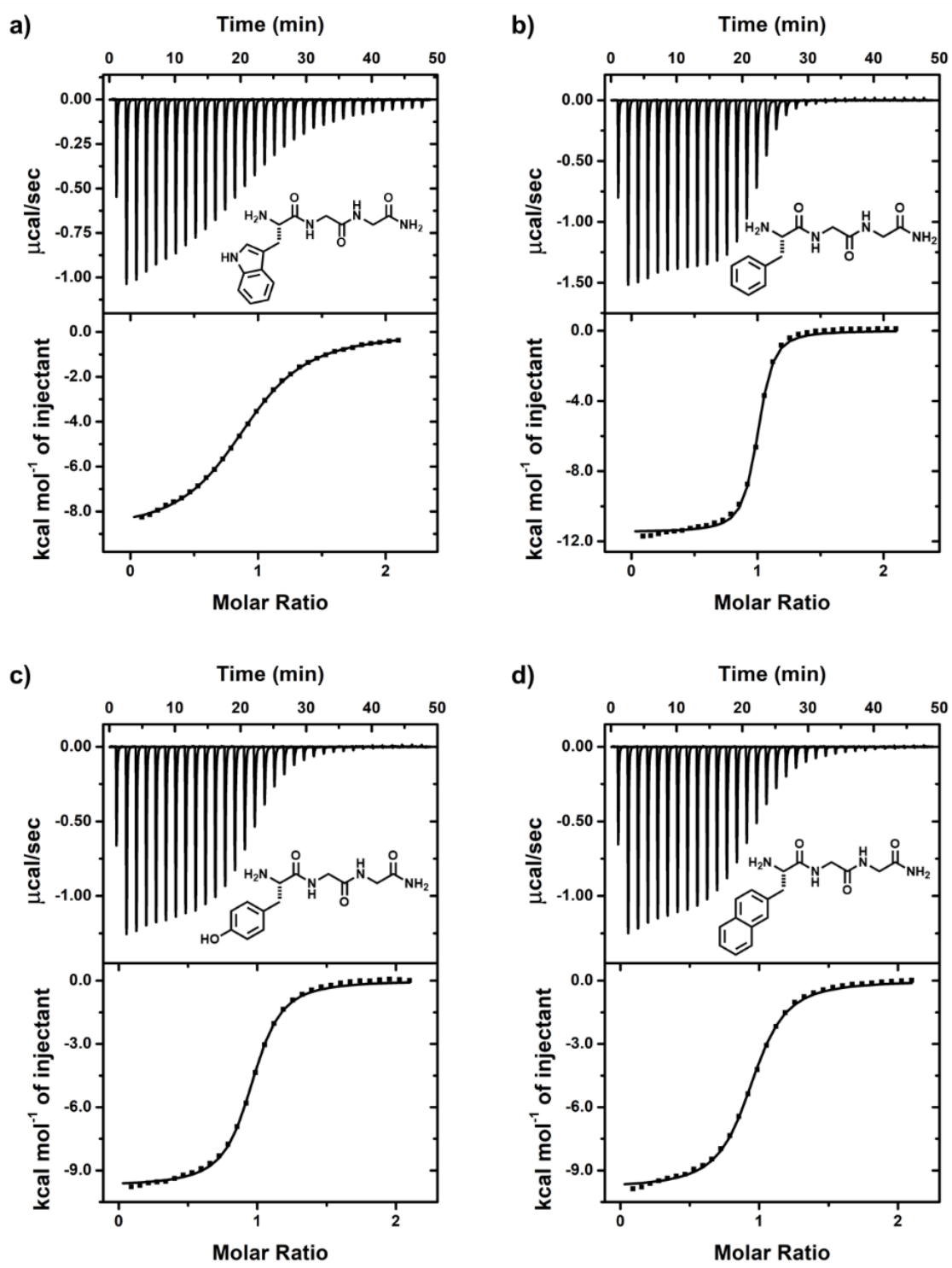

Figure S22 Representative ITC titration curves obtained by titrating a) WGG; b) FGG; c) YGG; d) NpGG into CB[7], respectively. All ITC experiments were repeated for 3 times. $(298 \mathrm{~K}, 10 \mathrm{mM}$ phosphate buffer, $\mathrm{pH}=7.0)$ 
Table S1 Thermodynamic data for homoternary complexation of aromatic tripeptides with $\mathrm{CB}[8]^{\mathrm{a}}$

\begin{tabular}{c|ccc|ccc|c}
\hline $\begin{array}{c}\text { model } \\
\text { peptide }\end{array}$ & $\begin{array}{c}K_{1} \\
\left(10^{4} \mathrm{M}^{-1}\right)\end{array}$ & $\begin{array}{c}\Delta H_{1} \\
\left(\mathrm{kcal} \mathrm{mol}^{-1}\right)\end{array}$ & $\begin{array}{c}-T \Delta S_{1} \\
\left(\mathrm{kcal} \mathrm{mol}^{-1}\right)\end{array}$ & $\begin{array}{c}K_{2} \\
\left(10^{4} \mathrm{M}^{-1}\right)\end{array}$ & $\begin{array}{c}\Delta H_{2} \\
\left(\mathrm{kcal} \mathrm{mol}^{-1}\right)\end{array}$ & $\begin{array}{c}-T \Delta S_{2} \\
\left(\mathrm{kcal} \mathrm{mol}^{-1}\right)\end{array}$ & $\mathrm{R}^{\mathrm{b}}$ \\
\hline F'GG & $66 \pm 4$ & $-12.6 \pm 0.4$ & $4.5 \pm 0.3$ & $0.94 \pm 0.09$ & $-6.7 \pm 0.5$ & $1.3 \pm 0.5$ & - \\
WGG & $27 \pm 5$ & $-10.6 \pm 0.1$ & $3.2 \pm 0.1$ & $2.5 \pm 0.1$ & $-7.8 \pm 0.5$ & $1.8 \pm 0.5$ & $2291 \pm 785$ \\
FGG & $32 \pm 3$ & $-10.6 \pm 0.1$ & $3.1 \pm 0.1$ & $25 \pm 1$ & $-13.2 \pm 0.1$ & $5.8 \pm 0.1$ & $109 \pm 27$ \\
YGG & $8 \pm 1$ & $-7.9 \pm 0.1$ & $1.2 \pm 0.1$ & $1.1 \pm 0.1$ & $-9.5 \pm 0.3$ & $3.9 \pm 0.3$ & $70 \pm 28$ \\
NpGG & $66 \pm 6$ & $-9.8 \pm 0.2$ & $1.8 \pm 0.2$ & $7.8 \pm 0.5$ & $-12.5 \pm 0.2$ & $5.8 \pm 0.2$ & $199 \pm 47$ \\
\hline
\end{tabular}

Note: a. All the above parameters were averaged with three replicates. b. The relative ratio of hetero- and homodimers shown as below:
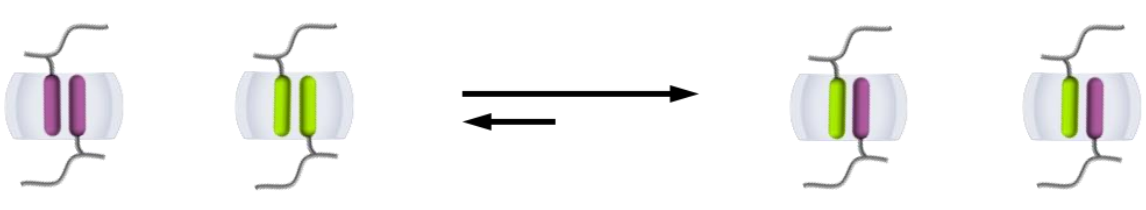

$$
\mathbf{R}=\frac{[\text { heterodimers }]}{[\text { homodimers }]}=\frac{\left(K_{1\left(\mathbf{F}^{\prime} \mathbf{G G}\right)} \cdot K_{a(\mathbf{X G G})}\right)^{2}}{\left(K_{1} K_{2}\right)_{\mathrm{F}^{\prime} \mathrm{GG}} \cdot\left(K_{1} K_{2}\right)_{\mathrm{XGG}}}
$$


Table S2 Thermodynamic data for binary complexation of aromatic tripeptides with $\mathrm{CB}[7]^{\mathrm{a}}$

\begin{tabular}{c|ccc}
\hline model peptide & $K\left(10^{4} \mathrm{M}^{-1}\right)$ & $\Delta H\left(\mathrm{kcal} \mathrm{mol}^{-1}\right)$ & $-T \Delta S\left(\mathrm{kcal} \mathrm{mol}^{-1}\right)$ \\
\hline WGG & $13 \pm 0.8$ & $-8.91 \pm 0.1$ & $1.9 \pm 0.1$ \\
FGG & $280 \pm 10$ & $-11.4 \pm 0.1$ & $2.6 \pm 0.1$ \\
YGG & $82 \pm 3$ & $-9.56 \pm 0.1$ & $1.5 \pm 0.1$ \\
NpGG & $58 \pm 3$ & $-9.64 \pm 0.2$ & $1.8 \pm 0.2$ \\
\hline
\end{tabular}

Note: a. All the above parameters were averaged with three replicates. 


\section{SI-5 Quantification of on-resin recognition by UV-Vis \& ${ }^{1} \mathrm{H}$ NMR}

(I) Quantitative analysis for on-resin recognition through UV measurements

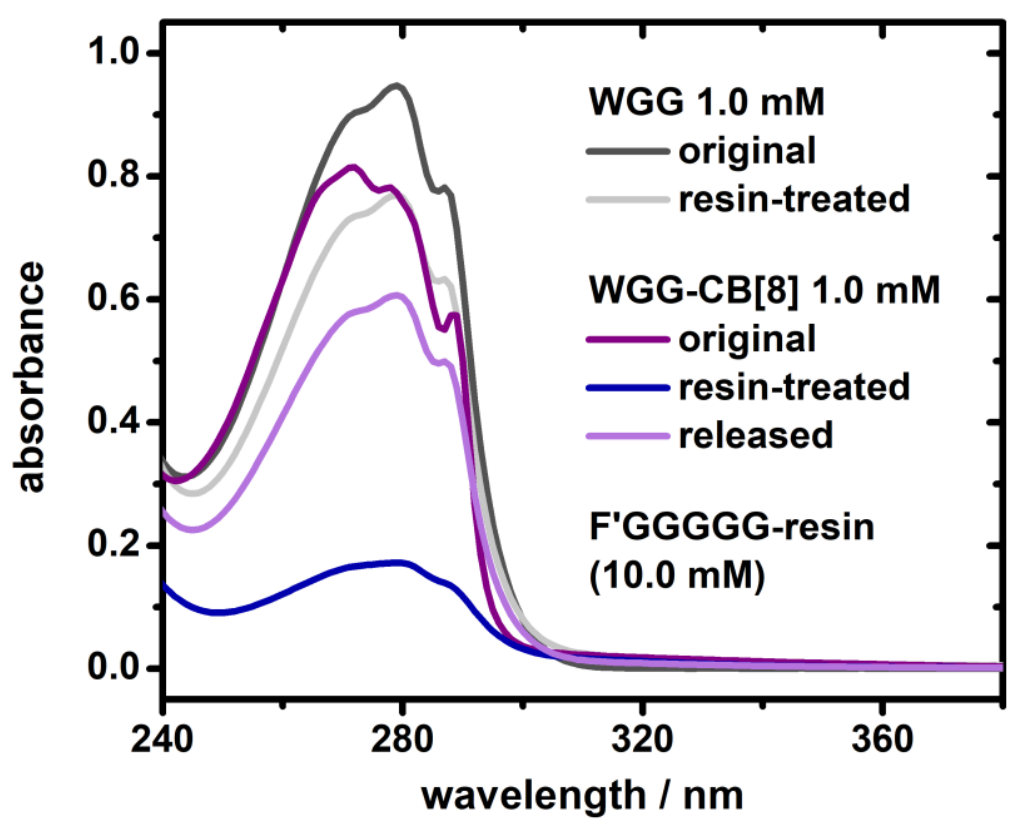

Figure S23 UV spectra for original (black) and resin-treated (grey) WGG, original (purple) and resin-treated (blue) WGG-CB[8] followed with competitive release (light purple) from F'GGGGG-resins by DMADA. All the solutions were prepared with $10 \mathrm{mM}$ phosphate buffer of $\mathrm{pH} 7.0$ in $\mathrm{D}_{2} \mathrm{O}$.

Table S3 UV absorbance at $279 \mathrm{~nm}$ for WGG and WGG-CB[8] at different states

\begin{tabular}{|c|c|c|c|c|c|}
\hline \multirow[b]{2}{*}{ state } & \multicolumn{2}{|c|}{ WGG } & \multicolumn{3}{|c|}{ WGG-CB[8] } \\
\hline & original & resin-treated & original & resin-treated & released \\
\hline repeat 1 & 0.9470 & 0.7695 & 0.7933 & 0.1805 & 0.6140 \\
\hline repeat 2 & 0.9473 & 0.7693 & 0.7938 & 0.1808 & 0.6143 \\
\hline repeat 3 & 0.9477 & 0.7690 & 0.7941 & 0.1808 & 0.6146 \\
\hline average & 0.9474 & 0.7693 & 0.7937 & 0.1807 & 0.6143 \\
\hline std dev & 0.0004 & 0.0003 & 0.0004 & 0.0002 & 0.0003 \\
\hline absorption & \multirow{2}{*}{\multicolumn{2}{|c|}{$\begin{array}{c}(0.9474-0.7693) / 0.9474 \\
\text { x } 100 \%=\mathbf{1 8 . 7 \%}\end{array}$}} & \multirow{2}{*}{\multicolumn{3}{|c|}{$\begin{array}{c}(0.7937-0.1807) / 0.7937 \\
\text { x } 100 \%=77.2 \%\end{array}$}} \\
\hline efficiency & & & & & \\
\hline
\end{tabular}


(II) Time-dependent investigations for on-resin recognition and release

a)

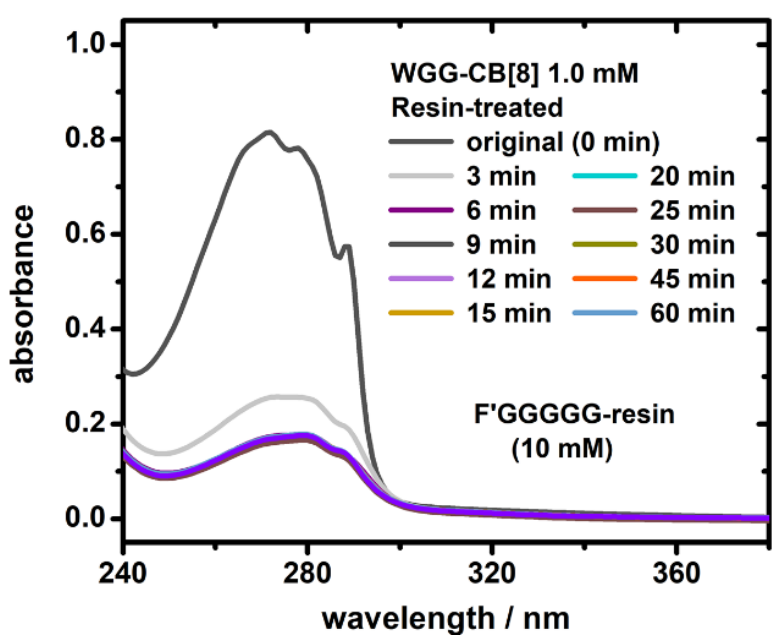

b)

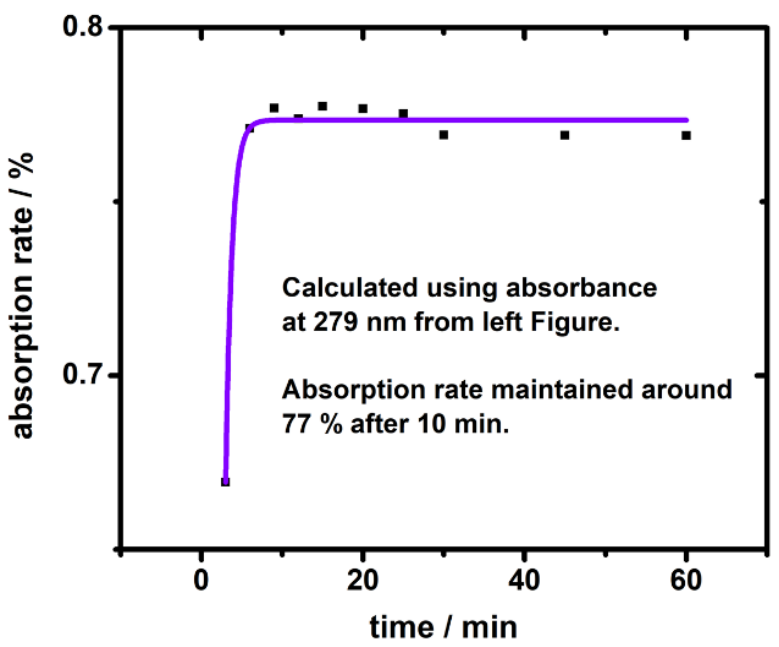

Figure S24 a) Time-dependent UV spectra of WGG-CB[8] treated with F'GGGGG-resins (3 - 60 min); b) plot and fitted curve for the absorption rate calculated upon UV absorbance at $279 \mathrm{~nm}$.

a)

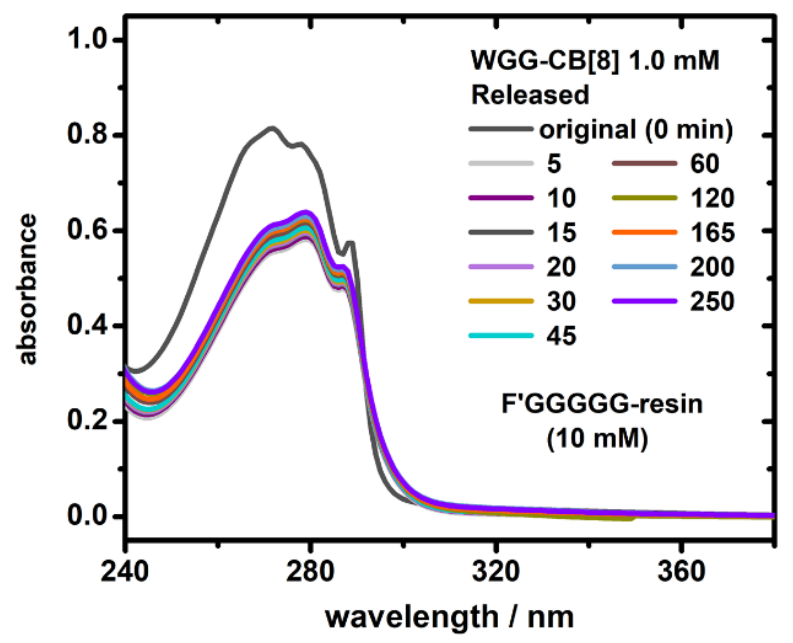

b)

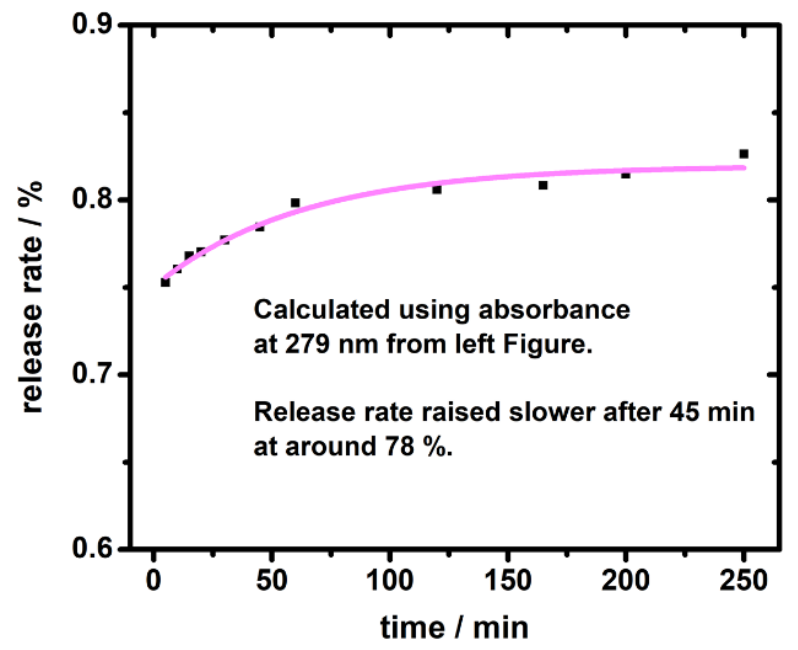

Figure S25 a) Time-dependent UV spectra of WGG-CB[8] released from F'GGGGG-resins (5 - 250 min); b) plot and fitted curve for the release rate curve calculated upon absorbance at $279 \mathrm{~nm}$.

As shown in Figure S25, WGG-CB[8] bound to F'GGGGG-resin rapidly and the absorption rate plateaued around $77 \%$ after $10 \mathrm{~min}$. In Figure S26, the release process happened slower than binding, however, most of WGG was released after around $45 \mathrm{~min}$ at a release rate of $78 \%$. After considering the practicability and proficiency of the on-resin recognition experiments, throughout this study, the resintreating time was set to 10 min while releasing time was $45 \mathrm{~min}$. 
(III) Multi-cycle on-resin recognition

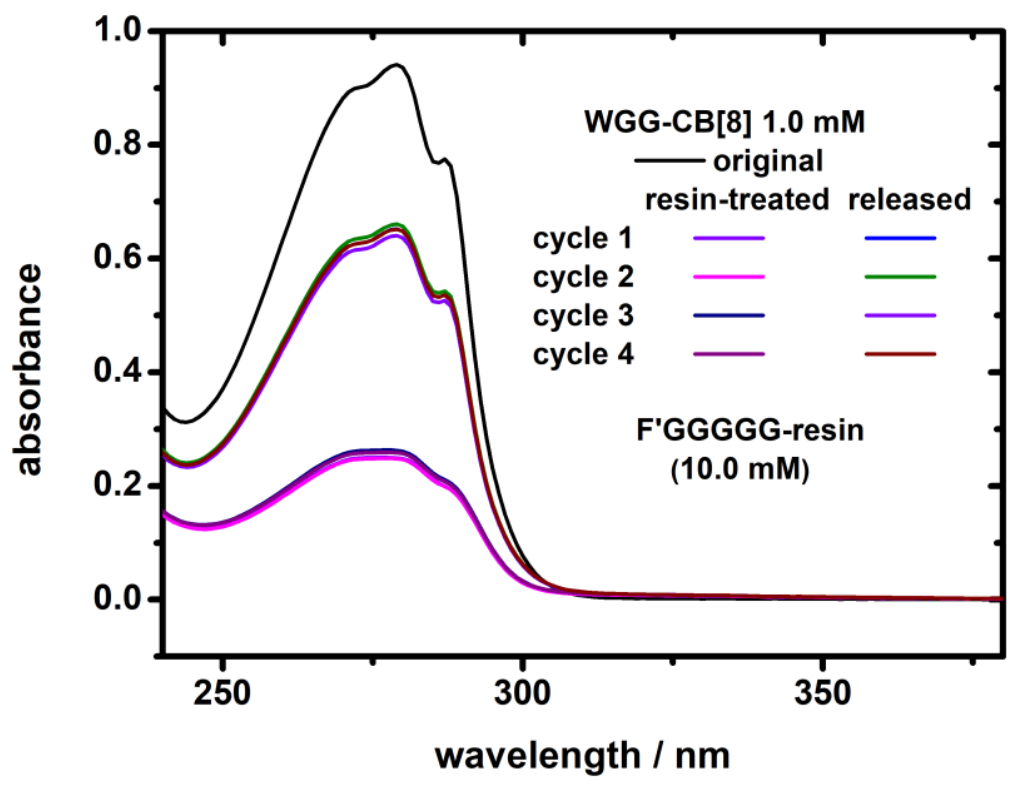

Figure S26 UV spectra of WGG-CB[8] (1.0 mM) before and after treated by F'GGGGG-resins (10 mM) for 4 consecutive cycles (phosphate buffer, $\mathrm{D}_{2} \mathrm{O}$ ).

Table S4 UV-Vis absorbance data at $279 \mathrm{~nm}$ for multi-cycle on-resin recognition.

\begin{tabular}{l|lllll}
\hline & $\begin{array}{l}\text { WGG-CB[8] } \\
\text { original (Abs) }\end{array}$ & $\begin{array}{l}\text { WGG-CB[8] } \\
\text { resin-treated } \\
\text { cycle 1 (Abs) }\end{array}$ & $\begin{array}{l}\text { WGG-CB[8] } \\
\text { resin-treated } \\
\text { cycle 2 (Abs) }\end{array}$ & $\begin{array}{l}\text { WGG-CB[8] } \\
\text { resin-treated } \\
\text { cycle 3 (Abs) }\end{array}$ & $\begin{array}{l}\text { WGG-CB[8] } \\
\text { resin-treated } \\
\text { cycle 4 (Abs) }\end{array}$ \\
\hline repeat 1 & 0.9383 & 0.2481 & 0.2465 & 0.2610 & 0.2572 \\
repeat 2 & 0.9412 & 0.2499 & 0.2478 & 0.2626 & 0.2578 \\
repeat 3 & 0.9431 & 0.2503 & 0.2500 & 0.2639 & 0.2605 \\
average & 0.9409 & 0.2494 & 0.2481 & 0.2625 & 0.2585 \\
std dev & 0.0024 & 0.0012 & 0.0018 & 0.0015 & 0.0018 \\
efficiency $(\%)$ & - & $\mathbf{7 3 . 4 9 \%}$ & $\mathbf{7 3 . 6 3 \%}$ & $\mathbf{7 2 . 1 0 \%}$ & $\mathbf{7 2 . 5 2 \%}$ \\
\hline
\end{tabular}

Note: The efficiency results for Figure $4 \mathrm{c}$ were calculated from Table S4 using the equation:

$$
\text { Efficiency } y_{\text {on-resin recognition }}=1-\frac{A b s_{\mathrm{WGG}-\mathrm{CB}[8] \text { resin-treated }}}{A b s_{\mathrm{WGG}-\mathrm{CB}[8] \text { original }}}
$$


(IV) On-resin selective isolation of aromatic peptides

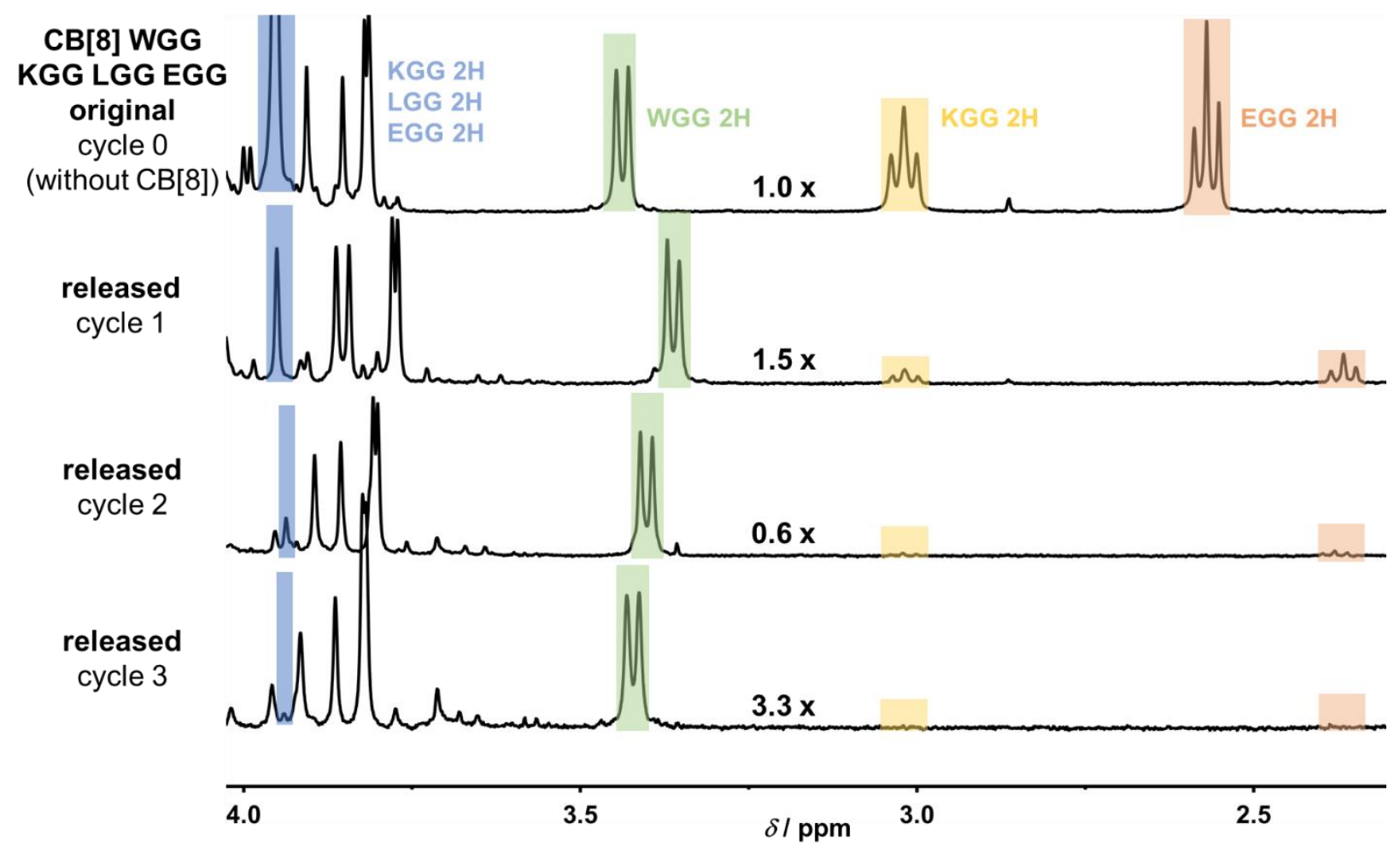

Figure $\mathbf{S 2 7}{ }^{1} \mathrm{H}$ NMR of on-resin selective isolation of aromatic peptides from mixed peptide mixture (WGG, KGG, EGG, LGG $1.0 \mathrm{mM}$, phosphate buffer, $\mathrm{D}_{2} \mathrm{O}$ ) over 3 cycles using F'GGGGG-resins (10 mM).

Table S5 ${ }^{1} \mathrm{H}$ NMR integration analysis for on-resin recognition within a peptide mixture

\begin{tabular}{c|ccccccccc}
\hline \multirow{2}{*}{} & \multicolumn{2}{|c}{ WGG } & \multicolumn{2}{c}{ KGG } & \multicolumn{2}{c}{ EGG } & KGG+LGG+EGG & LGG \\
\cline { 2 - 9 } & area & percentage (\%) & area & $\begin{array}{c}\text { percentage } \\
(\%)\end{array}$ & area & $\begin{array}{c}\text { percentage } \\
(\%)\end{array}$ & total area & area & $\begin{array}{c}\text { percentage } \\
(\%)\end{array}$ \\
\hline cycle 0 & 1000.0 & 24.5 & 919.6 & 22.6 & 1129.5 & 27.7 & 3078.1 & 1029 & 25.2 \\
cycle 1 & 1000.0 & 72.7 & 108.8 & 7.9 & 175.3 & 12.7 & 376.1 & 92 & 6.7 \\
cycle 2 & 1000.0 & 86.7 & 25.6 & 2.2 & 40.5 & 3.5 & 153.2 & 87.1 & 7.6 \\
cycle 3 & 1000.0 & 95.0 & 16.4 & 1.6 & 25.4 & 2.4 & 52.7 & 10.9 & 1.0 \\
\hline
\end{tabular}

Note: All the above results of percentages were shown in Figure 4d. 
(V) On-resin recognition on GGGGGG resin

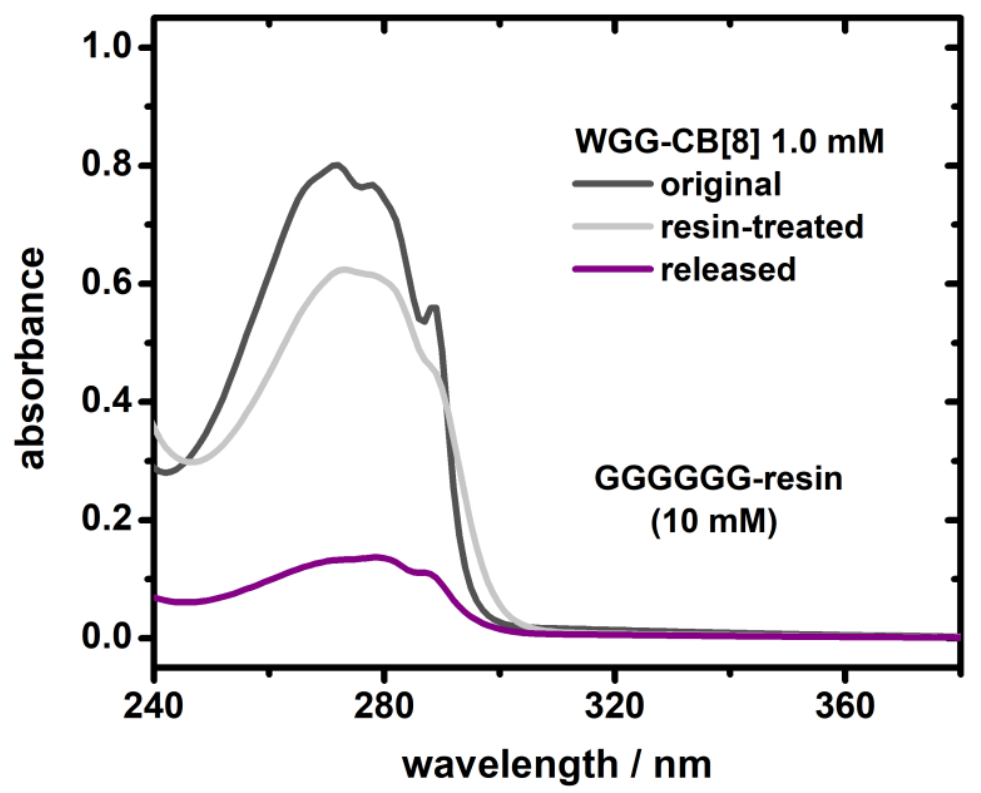

Figure S28 UV spectra of WGG-CB[8] $(1.0 \mathrm{mM})$ before, after treated by and released from GGGGGGresins $(10 \mathrm{mM})$ (phosphate buffer, $\left.\mathrm{D}_{2} \mathrm{O}\right)$.

As shown in Figure S29, by using the same ChemMatrix resin but with different peptide GGGGGG other than F'GGGGG loaded, WGG-CB[8] complexes were almost not absorbed to the resin. The minimal amount of released signal may come from non-specific absorption. This confirmed that the absorption of WGG-CB[8] to F'GGGGG-resins was achieved by host-enhanced polar- $\pi$ interactions. 


\section{References}

[1] J. Kim, I.-S. Jung, S.-Y. Kim, E. Lee, J.-K. Kang, S. Sakamoto, K. Yamaguchi, K. Kim, J. Am. Chem. Soc. 2000, 122, 540-541. 


\title{
Quantitative Heterodimerization of Aromatic Peptides through Host-Enhanced Polar- $\pi$ Interactions for On-Resin Recognition
}

\author{
Xiaoyi Chen ${ }^{\dagger}$, Zehuan Huang ${ }^{\dagger}$, Guanglu Wu, Kamil Sokłolowski, Katherine King, Jade A. McCune,
} and Oren A. Scherman*

Abstract: Peptide dimerization plays an important role in
both natural and artificial supramolecular systems. A ma-
jor challenge to date is the quantitative heterodimerization
of two peptides without formation of homodimers. Here,
we employ a macrocyclic host to simultaneously encap-
sulate a canonical aromatic peptide and a non-canonical
perfluorophenylalanine-containing peptide through polar- $\pi$
interactions, thus forming an unprecedented new series of
heteropeptide dimers with high binding affinity. This new
peptide heterodimerization was applied to on-resin recogni-
tion and separation of aromatic peptides in a peptide mix-
ture exhibiting over 95\% isolation purity. This research un-
veils a generic approach to exploit quantitative heteropep-
tide dimers for the design of supramolecular (bio)systems.
Dimerization of peptides through covalent bonding or non-covalent association is of great importance in the structural design and functional control of natural ${ }^{[1-3]}$ and artificial $^{[4-6]}$ self-assembly. Covalent conjugation requires elaborate reactions to form static, strong covalent bonds between peptides, ${ }^{[7-10]}$ while non-covalent peptide dimerization is more facile and versatile, ${ }^{[11-14]}$ on account of their dynamic and reversible nature. However, the relatively weak association and low specificity of non-covalent dimers have limited their use in aqueous systems. To address this problem, many macrocyclic hosts have been employed to encapsulate hydrophobic residues of peptides in their nanocavities, thus enhancing the overall binding strength of non-covalent peptide dimers. ${ }^{[4,6]}$

On account of high binding affinity and the wide scope of guests, cucurbit[n]uril ( $\mathrm{CB}[\mathrm{n}])$-mediated host-guest complexes are ideal systems to bind peptides. ${ }^{[15-20]}$ Urbach et al. found that two FGG tripeptides can be complexed with $\mathrm{CB}[8]$ to form a peptide homodimer, exhibiting high binding strength $\left(K \approx 10^{11} \mathrm{M}^{-2}\right){ }^{[15]}$ This type of homodimer has been adopted as a versatile building block in the design and fabrication of supramolecular oligomers ${ }^{[21]}$ and

\footnotetext{
* Xiaoyi Chen ${ }^{\dagger}$, Zehuan Huang ${ }^{\dagger}$, Guanglu Wu, Kamil Sokłolowski, Katherine King, Jade A. McCune, and Oren A. Scherman Melville Laboratory for Polymer Synthesis,

Yusuf Hamied Department of Chemistry

University of Cambridge

Lensfield Road, Cambridge, CB2 1EW, UK

E-mail: oas23@cam.ac.uk

$\dagger$ These authors contributed equally to this work.
}

polymers, ${ }^{[22,23]}$ supramolecular hydrogels, ${ }^{[24,25]}$ and protein/peptide assemblies. ${ }^{[26,27]}$ Although significant strides have been made, a major challenge to date remains quantitative dimerization of two different peptides without forming any homodimers.

Herein, we propose a general strategy to employ a macrocyclic host to mediate heterodimerization of a canonical aromatic peptide and a non-canonical perfluorophenylalanine (F')-containing peptide, Figure 1a. Recently, we reported that an electron-poor perfluorophenyl first guest and an electron-rich phenyl second guest can exclusively form a heteroternary complex within the $\mathrm{CB}$ [8] cavity, yielding a host-enhanced polar $-\pi$ interaction. ${ }^{[28]}$ The incorporation of a non-canonical F' into a peptide sequence (e.g. F'GG) could act as a first guest, forming a more favorable 1:1 complex with $\mathrm{CB}[8]$ on account of the electrostatic repulsion that exists within its 2:1 complex. On this basis, subsequent association of various aromatic peptides (e.g. WGG) with the
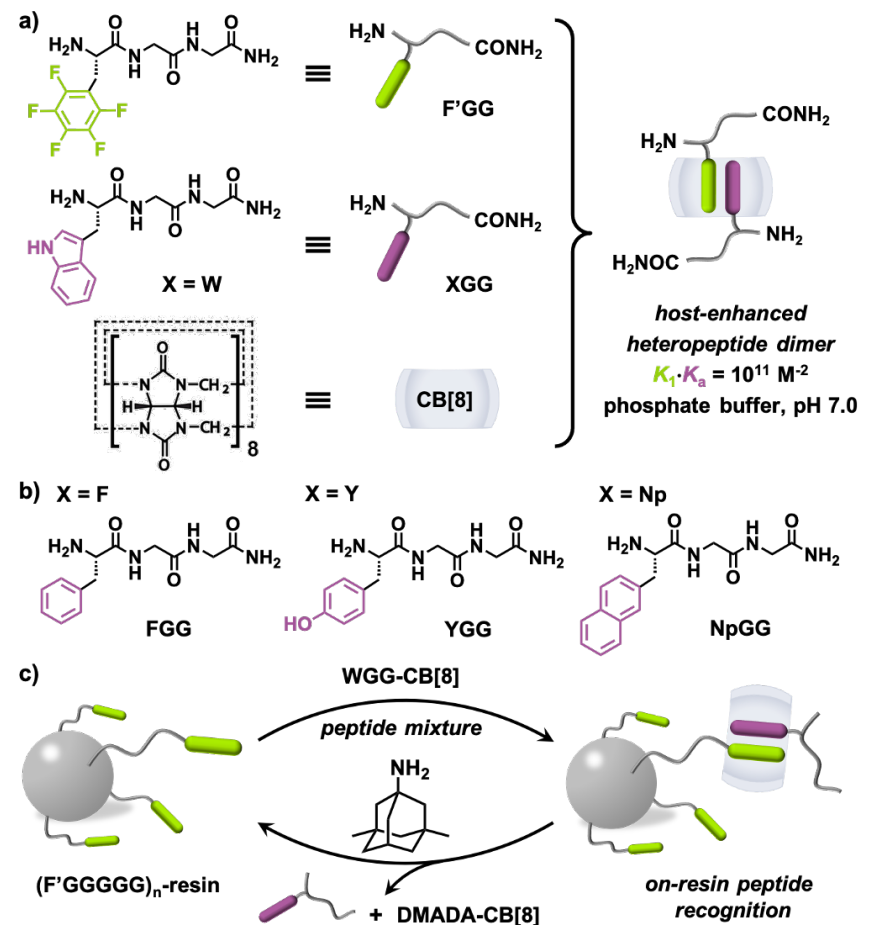

Figure 1 Schematic representations of a) host-enhanced heteropeptide dimer formed from F'GG, WGG, and $\mathrm{CB}[8]$; b) molecular structures of FGG, YGG, NpGG; c) on-resin peptide recognition through interfacial $\mathrm{CB}[8]$-mediated heteropeptide dimerization. 
a)

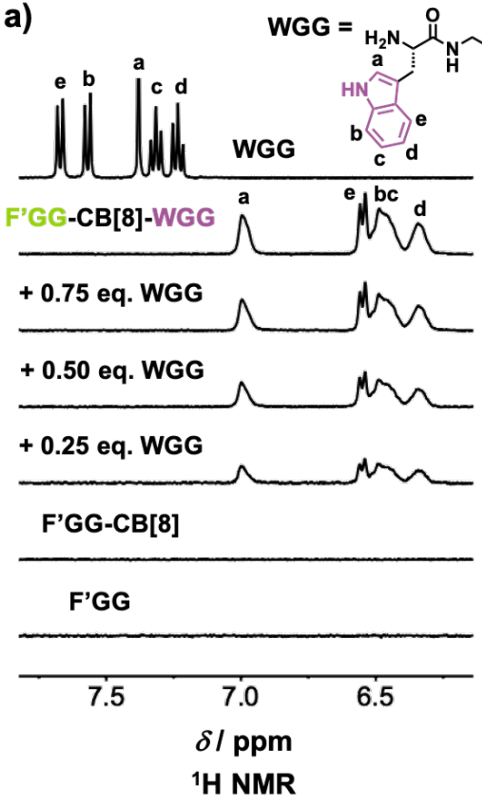

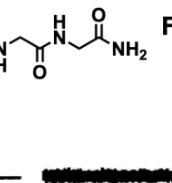
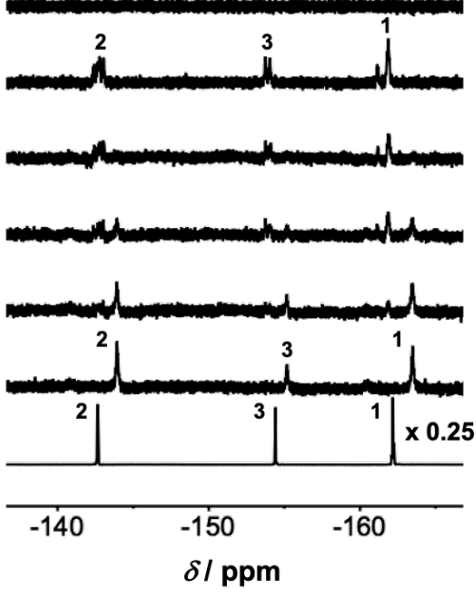

${ }^{19}$ F NMR b)
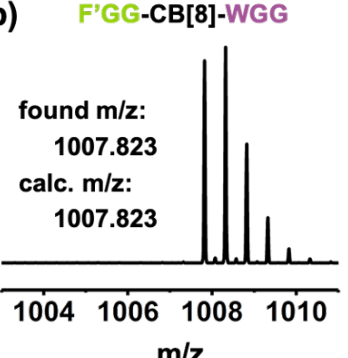

$\mathrm{m} / \mathbf{z}$

F'GG-CB[8]-YGG

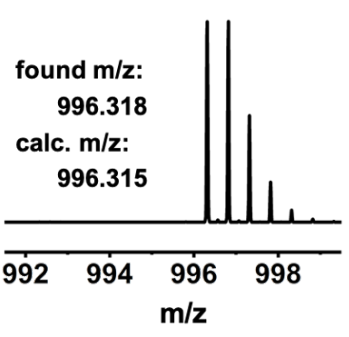

HR ESI-MS

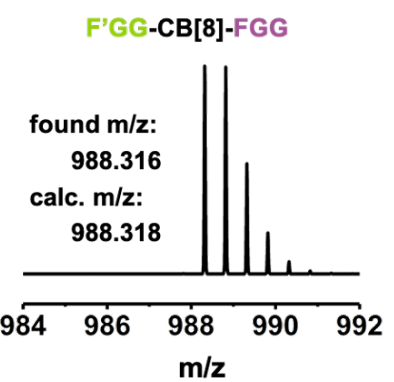

F'GG-CB[8]-NpGG

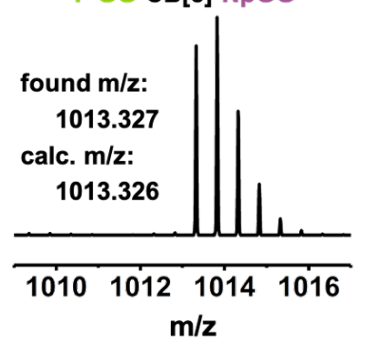

Figure 2 a) ${ }^{1} \mathrm{H}$ and ${ }^{19} \mathrm{~F}$ NMR spectra $\left(\mathrm{D}_{2} \mathrm{O}, 298 \mathrm{~K}\right)$ obtained through titration of WGG (16.0 mM) into F' GG-CB[8] (1.0 mM); b) HR ESIMS spectra of heteropeptide dimers $\left(\mathrm{H}_{2} \mathrm{O}, 1.0 \mathrm{mM}\right)$ of WGG, FGG, YGG, NpGG with 1:1 complex of F'GG-CB[8].

1:1 complex (e.g. F'GG-CB[8]) allows access to a new type of host-enhanced heteropeptide dimer with increased binding strength, Figure 1a.

As a proof of concept, five model aromatic tripeptides containing L-perfluorophenylalanine (F'GG), L-tryptophan (WGG), L-phenylalanine (FGG), L-tyrosine (YGG), and L(2-naphthyl)alanine (NpGG) at the $\mathrm{N}$ termini were designed and prepared, Figure 1a-b. We envisioned that an equimolar mixture of F'GG, $\mathrm{CB}[8]$, and XGG should result in an exclusive heteropeptide dimer instead of an equilibrium mixture also containing homodimers. Two series of tripeptides (GGX, GXG), containing aromatic amino acids either at the $\mathrm{C}$ termini or in the middle of the chain, were synthesized to investigate diversity of the second guest and its distance away from the positive charge, Chart S1. After elucidating the thermodynamics of the system, we applied this heterodimerization approach to achieve on-resin recognition and isolation ${ }^{[29]}$ of aromatic peptides from a peptide mixture exhibiting high efficiency and selectivity, Figure 1c.

Extensive ${ }^{1} \mathrm{H}$ and ${ }^{19} \mathrm{~F}$ NMR titrations were performed to study the formation of heteropeptide dimers with the model tripeptides, Figure 2a and Figures S1-S16. Titration of free WGG into a 1:1 mixture of F'GG-CB[8] resulted in a gradual appearance of the indole protons at 6.25-7.10 ppm, Figure $2 \mathrm{a}$. On account of the shielding effect from the CB[8] cavity, the indole proton peaks exhibited clear upfield shifts compared to free WGG. This suggests that the indole group in the F' GG-CB[8]-WGG complex is located in a new chemical environment different from that of unbound WGG. This titration process was also monitored by ${ }^{19} \mathrm{~F}$ NMR, Figure 2a; a new group of fluorine peaks gradually appeared, while those fluorine peaks in the binary complex of F'GG-CB[8] disappeared. These results strongly indicated the formation of a new heteropeptide dimer, F'GG-CB[8]-WGG. Additionally, equivalent mixtures of F' GG, CB[8] and XGG were prepared and characterized by high-resolution ESI-MS, Figure $2 b$. All of the ion peaks for F'GG-CB[8]-XGG were found with their calculated $\mathrm{m} / \mathrm{z}$ values, which confirmed successful preparation of the heteropeptide dimers.

Isothermal titration calorimetry (ITC) was employed to study binding thermodynamics of the heteropeptide dimers, Figure 3. Titration of F'GG (3.0 mM) into CB[8] (0.1 mM) led to a stepwise binding curve with both a sharp and a mild transition at the molar ratio of 1.0 and 2.0, respectively, Figure 3a. This binding curve is indicative of negative cooperativity as previously reported. ${ }^{[28,30]}$ This is on account of the strong electrostatic repulsion between two electron-poor perfluorophenyl motifs within a 2:1 complex, key to favoring 1:1 complexation and allowing subsequent binding of electron-rich aromatic peptides.

Four aromatic tripeptides (XGG, $\mathrm{X}=\mathrm{W}, \mathrm{F}, \mathrm{Y}, \mathrm{Np}$; $3.0 \mathrm{mM}$ ) were further titrated into a 1:1 complex of F'GG$\mathrm{CB}[8](0.2 \mathrm{mM})$ to investigate their binding as second guests within heteropeptide dimers. These titrations resulted in a series of binding curves with a clear transition at the molar ratio of 1.0 (Figure 3b), indicating the successful incorporation of XGG into F'GG-CB[8]. Table 1 summarizes the thermodynamic parameters for the complexation of XGG with F'GG-CB[8]. WGG, FGG, YGG, and NpGG exhibited high binding affinity with $K_{\mathrm{a}}$ values above $10^{4} \mathrm{M}^{-1}$, assuring the thermodynamic stability of heteropeptide dimers.

YGG (Figure 3b, green dataset) exhibited a relatively low secondary binding affinity as the para-substituted hydroxyl group may reduce the release of high-energy water, ${ }^{[31]}$ thus decreasing the enthalpic contribution and weakening the sec- 
a)

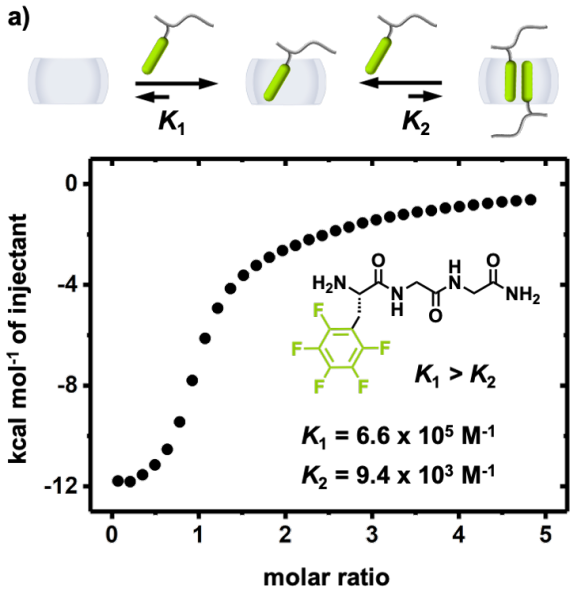

b)

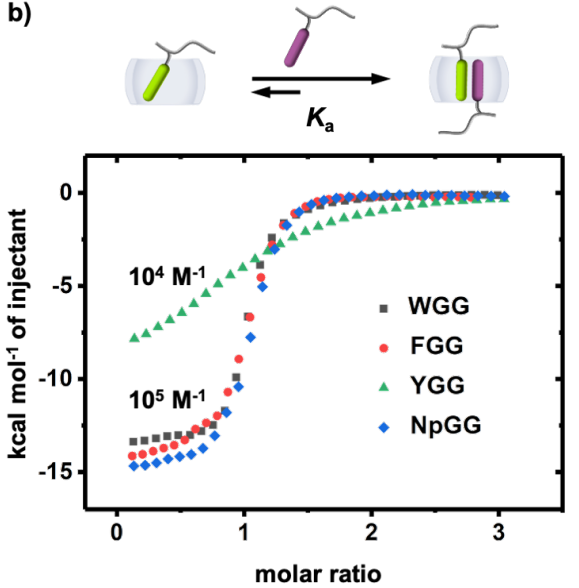

c)

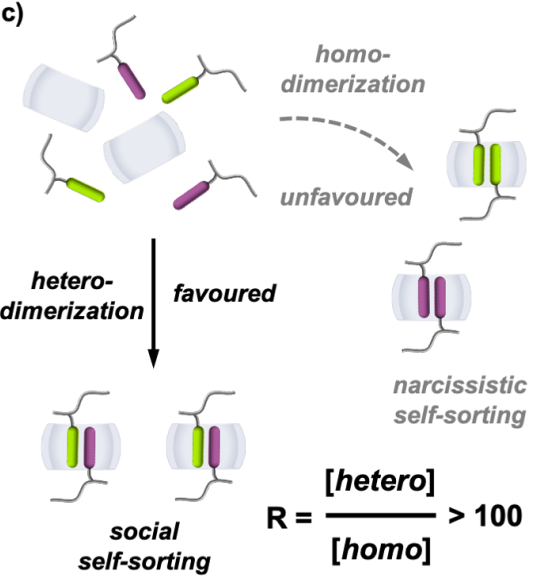

Figure 3 ITC titration plots (10 mM phosphate buffer, pH 7.0, $298 \mathrm{~K}$ ) of a) F' GG (3.0 mM) into CB[8] (0.1 mM); b) WGG, FGG, YGG, NpGG (3.0 mM) into F'GG-CB[8] (0.2 mM); and c) self-sorting mechanism behind host-enhanced heteropeptide dimerization.

ondary association. Nevertheless, the overall binding constants $\left(K_{1} \cdot K_{\mathrm{a}}\right)$ for the heteropeptide dimers F'GG-CB[8]XGG are all higher than $10^{10} \mathrm{M}^{-2}$. This shows significant enhancement compared to their parent dimers (e.g. F'GGWGG $\left.{ }^{[12,13]} K_{\text {dimer }} \approx 1 \mathrm{M}^{-1}\right)$. Notably, no secondary association of non-aromatic tripeptides (KGG, EGG, LGG) was observed, which unambiguously highlights the binding selectivity for aromatic over non-aromatic peptides.

We posited that peptide sequences with asymmetric $\mathrm{C}$ and $\mathrm{N}$ termini may influence the formation of host-enhanced heteropeptide dimers. To understand the effect of position, the secondary associations of GXG and GGX (X =W, F, Y, $\mathrm{Np}$ ) with the 1:1 complex of F'GG-CB[8] were studied by ITC. Shifting the aromatic residue from the $\mathrm{N}$ to $\mathrm{C}$ terminus led to a substantial decrease in second binding constants by nearly one order of magnitude, Table 1 . This is on account of the increased distance between the positive charge at the $\mathrm{N}$ terminus and the aromatic motif. This weakens ion-dipole interactions at the $\mathrm{CB}[8]$ portal and destabilizes the aromatic residue within the cavity, thus reducing the secondary binding affinity. This is exemplified by an extreme case where no secondary binding of GGY with F'GG-CB[8] was observed. Seven new, derivatized heteropeptide dimers, F'GG-CB[8]GXG (X = W, F, Y, Np) and F'GG-CB[8]-GGX (X = W, F, $\mathrm{Np}$ ), were readily formed, greatly expanding the scope of host-enhanced heteropeptide dimers.

The mechanism behind the formation of the heteropeptide dimers is attributed to thermodynamic social selfsorting, Figure 3c, consistent with our previous report. ${ }^{[28]}$ Two dimerization pathways exist in an equimolar mixture of F'GG, CB[8] and XGG: social (heteropeptide dimers) and narcissistic (homopeptide dimers) self-sorting. Comparison with homopeptide dimerization (Table S1) revealed a substantial preference for social self-sorting with a relative ratio of hetero- and homopeptide dimers, $\mathrm{R}>100$. Therefore, host-enhanced heteropeptide dimers are dominant in this complex system, enabling them as promising building blocks in functional supramolecular (bio)systems.
After fully elucidating the mechanism, we further demonstrated the utility of host-enhanced heteropeptide dimers in an interfacial self-assembled system to achieve on-resin recognition and isolation of aromatic peptides, Figure 1c. Figure $4 \mathrm{a}$ showed a standard operating process for on-resin recognition. A buffered solution of peptides and $\mathrm{CB}[8]$ was mixed with F'GGGGG-functionalized ChemMatrix resins for $10 \mathrm{~min}$ at room temperature with rigorous shaking. Through host-enhanced heteropeptide dimerization, aromatic peptides (XGG) were immediately bound onto the resins to form F'GGGGG-CB[8]-XGG at the interface. The residual solution of the peptide mixture was removed, and then a buffered solution of memantine hydrochloride (DMADA) was added and thoroughly mixed with resins for

Table 1 Overview of thermodynamic data for complexation of aromatic tripeptides with the $1: 1$ complex of F'GG-CB[8] ${ }^{a}$

\begin{tabular}{cccc}
\hline $\begin{array}{c}\text { model } \\
\text { peptide }\end{array}$ & $\begin{array}{c}K_{\mathrm{a}} \\
\left(10^{4} \mathrm{M}^{-1}\right)\end{array}$ & $\begin{array}{c}\Delta H_{\mathrm{a}} \\
\left(\mathrm{kcal} \mathrm{mol}^{-1}\right)\end{array}$ & $\begin{array}{c}-T \Delta S_{\mathrm{a}} \\
\left(\mathrm{kcal} \mathrm{mol}^{-1}\right)\end{array}$ \\
\hline WGG & $46 \pm 5$ & $-13.3 \pm 0.2$ & $5.6 \pm 0.2$ \\
GWG & $10 \pm 1$ & $-11.9 \pm 0.1$ & $5.0 \pm 0.1$ \\
GGW & $9 \pm 1$ & $-14.5 \pm 0.3$ & $7.8 \pm 0.4$ \\
FGG & $36 \pm 3$ & $-14.4 \pm 0.4$ & $6.8 \pm 0.3$ \\
GFG & $5.8 \pm 0.3$ & $-9.1 \pm 0.4$ & $2.6 \pm 0.4$ \\
GGF & $2.2 \pm 0.1$ & $-7.5 \pm 0.2$ & $1.5 \pm 0.2$ \\
YGG & $3.0 \pm 0.5$ & $-9.6 \pm 0.1$ & $3.6 \pm 0.2$ \\
GYG & $0.40 \pm 0.03$ & $-4.7 \pm 0.2$ & $-0.2 \pm 0.3$ \\
GGY & $-{ }^{b}$ & $-{ }^{b}$ & $-{ }^{b}$ \\
NpGG & $38 \pm 2$ & $-14.6 \pm 0.2$ & $7.0 \pm 0.2$ \\
GNpG & $17 \pm 1$ & $-12.7 \pm 0.4$ & $5.6 \pm 0.4$ \\
GGNp & $9 \pm 1$ & $-12.0 \pm 0.1$ & $5.2 \pm 0.1$ \\
KGG & $-{ }^{b}$ & $-{ }^{b}$ & $-{ }^{b}$ \\
EGG & $-{ }^{b}$ & $-{ }^{b}$ & $-{ }^{b}$ \\
LGG & $-{ }^{b}$ & $-{ }^{b}$ & $-{ }^{b}$
\end{tabular}

Note: ${ }^{a}$ All these parameters were averaged with three replicates. ${ }^{b}$ These values were too low to be detected by ITC as $K_{\mathrm{a}}<10^{2} \mathrm{M}^{-1}$. 
a)

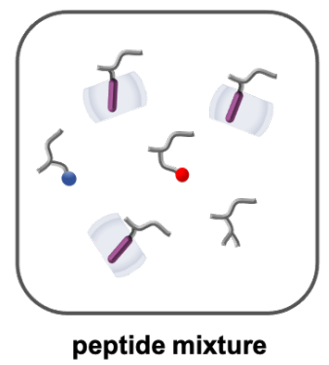

b)

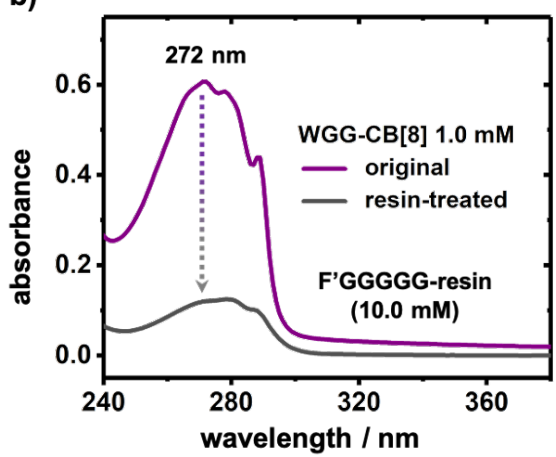

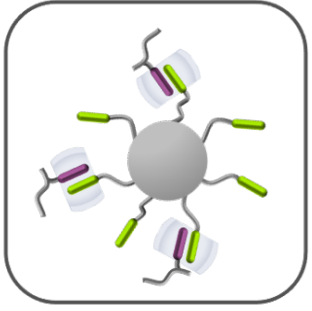

on-resin recognition c)

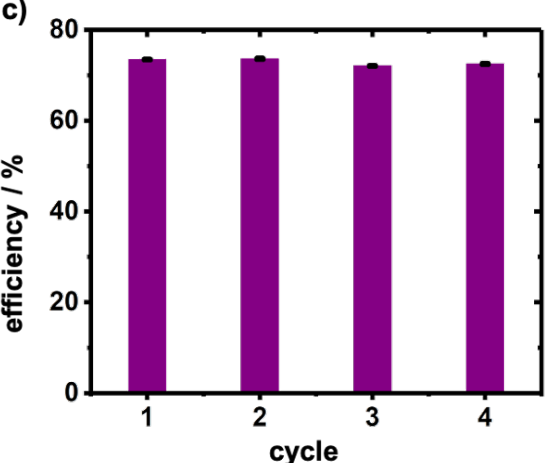

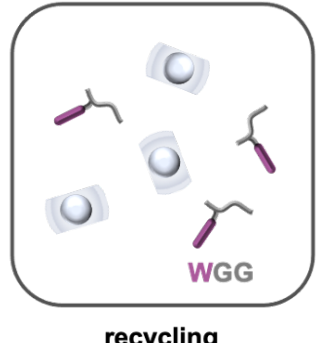

recycling

d)

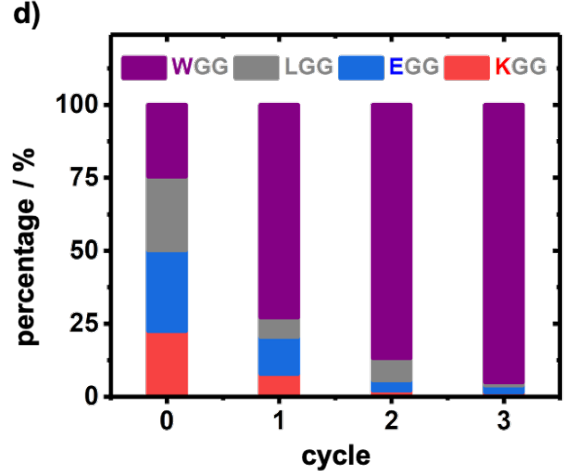

Figure 4 a) Schematic representation for on-resin recognition and separation of an aromatic peptide from a mixture containing nonaromatic peptides; b) UV spectra of WGG-CB[8] solution (1.0 mM) before and after treatment with F'GGGGG-resins (10.0 mM); histograms of c) recycling efficiency of continuous on-resin recognition with WGG and d) percentage of WGG obtained after multi-cycle isolation.

45 min. DMADA acts as a strong, competitive guest to replace the $\mathrm{CB}[8]$ host from the heteropeptide dimers, ensuring the release of the bound aromatic peptides and regenerating the functionalized resins.

To test the absorption efficiency of on-resin recognition, $\mathrm{UV}$ experiments were performed to measure the amount of aromatic peptides present before and after on-resin treatment, Figure 4b. A typical experiment involved mixing an aqueous buffer solution of WGG-CB[8] (1.0 mM) with the functionalized resin $(10.0 \mathrm{mM})$ for $10 \mathrm{~min}$. The UV spectra of the original and resin-treated solutions of WGG-CB[8] were obtained in the linear absorption range $(0.2-0.8)$. The absorption intensity of the resin-treated solution of WGG$\mathrm{CB}$ [8] (grey) showed a substantial decrease compared to that of the original one (purple), Figure 4b. The absorption efficiency for on-resin recognition was calculated to be as high as $77 \%$. By contrast, the efficiency for recognition with free WGG through pure polar- $\pi$ interactions and physical absorption is only $19 \%$, Table S3. The absorbed WGG was released and recycled through competitive replacement by DMADA within $45 \mathrm{~min}$, Figure S24. The residual DMADA and DMADA-CB[8] can be readily removed through liquid-phase chromatography. These results elucidate the key role of host-enhanced heteropeptide dimerization in on-resin recognition.

Multi-cycle on-resin recognition was performed to evaluate the recyclability of the F'GGGGG-functionalized resins, Figure 4c. Recognition-regeneration experiments on WGG$\mathrm{CB}[8]$ were repeated for four cycles using the same batch of resins. The on-resin absorption efficiency maintained above $75 \%$, Figure 4c. This is on account of the complete release of WGG by competitive replacement without any residue accumulation. These results confirm successful regeneration of the F'GGGGG-functionalized resins, endowing the whole process with high sustainability for practical applications. We further investigated selective isolation of aromatic peptides through recognition-release experiments over three cycles, Figure 4d, using a peptide mixture of WGG, KGG, EGG, and LGG ([XGG] $=1.0 \mathrm{mM}$ ) in the presence of $1.0 \mathrm{mM} \mathrm{CB}[8]$. The ratio of WGG (purple column) within the XGG mixture is significantly increased from $25.7 \%$ to $95.0 \%$ after three on-resin treatment cycles, Figure $4 \mathrm{~d}$. This demonstrates high isolation purity for aromatic peptides through on-resin heteropeptide dimerization.

In conclusion, we have successfully developed a new type of host-enhanced heteropeptide dimerization in aqueous media. Through $\mathrm{CB}[8]$-mediated polar- $\pi$ interactions, the binding affinity between aryl and perfluorophenyl groups from two different peptides is significantly enhanced with a $K_{\mathrm{a}}$ up to $10^{5} \mathrm{M}^{-1}$ and a $K_{1} \cdot K_{\mathrm{a}}$ up to $10^{11} \mathrm{M}^{-2}$, ensuring exclusive formation of heteropeptide dimers. To demonstrate the utility of these heterodimers, the solutionphase host-guest complex (F'GG-CB[8]-XGG) was transferred to a solid-liquid interface achieving on-resin recognition and isolation of aromatic peptides from a mixture with non-aromatic peptides, exhibiting over $73 \%$ absorption efficiency and $95 \%$ isolation purity. This generic approach will 
enable accumulation and separation of aromatic-abundant biomacromolecules in practical applications. We anticipate that this work will inspire renewed interest and research into the formation of heteropeptide dimers and their exploitation as versatile building blocks in supramolecular chemistry and the life sciences.

\section{Acknowledgement}

X.C. acknowledges CDT and EPSRC Grant (EP/R512461/1) for financial support. Z.H. thanks Marie Skłodowska-Curie Fellowship (845640). G.W., K.S., K.K, J.A.M. \& O.A.S. acknowledges ERC consolidator grant (CAM-RIG, 726470) and EPSRC Programme Grant (NOtCH, EP/L027151/1). We thank Daniel J. Whitaker, Stephen O'Neill, and Jiaxuan Li for their kind help.

\section{Conflict of Interest}

The authors declare no conflict of interest.

\section{Keywords}

heterodimers, peptides, host-guest interactions, cucurbit[n]urils, supramolecular chemistry

\section{References}

[1] X.-L. He, D.-C. Chow, M. M. Martick, K. Christopher Garcia, Science 2001, 293, 1657-1662.

[2] M. E. Call, J. R. Schnell, C. Xu, R. A. Lutz, J. J. Chou, K. W Wucherpfennig, Cell 2006, 127, 355-368.

[3] M. Schmidt, A. Rohou, K. Lasker, J. K. Yadav, C. Schiene-Fischer, M. Fändrich, N. Grigorieff, Proc. Natl. Acad. Sci. U.S.A. 2015, 112, 11858-11863.

[4] D. A. Uhlenheuer, K. Petkau, L. Brunsveld, Chem. Soc. Rev. 2010, 39, 2817-2826.

[5] Q. Luo, C. Hou, Y. Bai, R. Wang, J. Liu, Chem. Rev. 2016, 116 , 13571-13632.

[6] Supramolecular Protein Chemistry, Assembly, Architecture and Application, (Ed.: P. B. Crowley), The Royal Society of Chemistry, 2021, P001-312.

[7] M. W. Hornef, K. Pütsep, J. Karlsson, E. Refai, M. Andersson, Nat. Immunol. 2004, 5, 836-843.

[8] A. Jiménez-Balsa, E. Pazos, B. Martínez-Albardonedo, J. L. Mascareñas, M. E. Vázquez, Angew. Chem. Int. Ed. 2012, 51, 88258829.
[9] W. Tang, M. L. Becker, Chem. Soc. Rev. 2014, 43, 7013-7039.

[10] B. F. Fisher, S. H. Hong, S. H. Gellman, J. Am. Chem. Soc. 2017, 139, 13292-13295.

[11] M. M. C. Bastings, T. F. A. de Greef, J. L. J. van Dongen, M. Merkx, E. W. Meijer, Chem. Sci. 2010, 1, 79-88.

[12] H. Zheng, J. Gao, Angew. Chem. Int. Ed. 2010, 49, 8635-8639.

[13] C. J. Pace, H. Zheng, R. Mylvaganam, D. Kim, J. Gao, Angew. Chem. Int. Ed. 2012, 51, 103-107.

[14] C. Zhang, M. Welborn, T. Zhu, N. J. Yang, M. S. Santos, T. Van Voorhis, B. L. Pentelute, Nat. Chem. 2016, 8, 120-128.

[15] L. M. Heitmann, A. B. Taylor, P. J. Hart, A. R. Urbach, J. Am. Chem. Soc. 2006, 128, 12574-12581.

[16] L. C. Smith, D. G. Leach, B. E. Blaylock, O. A. Ali, A. R. Urbach, J. Am. Chem. Soc. 2015, 137, 3663-3669.

[17] S. Sonzini, A. Marcozzi, R. J. Gubeli, C. F. van der Walle, P. Ravn, A. Herrmann, O. A. Scherman, Angew. Chem. Int. Ed. 2016, 55, 14000-14004.

[18] Z. Hirani, H. F. Taylor, E. F. Babcock, A. T. Bockus, C. D. Varnado Jr, C. W. Bielawski, A. R. Urbach, J. Am. Chem. Soc. 2018, 140, 12263-12269.

[19] H. Wang, Y.-Q. Yan, Y. Yi, Z.-Y. Wei, H. Chen, J.-F. Xu, H. Wang, Y. Zhao, X. Zhang, CCS Chem. 2020, 2, 739-748.

[20] Y.-H. Liu, Y.-M. Zhang, H.-J. Yu, Y. Liu, Angew. Chem. Int. Ed. 2021, 60, 3870-3880.

[21] M. Ramaekers, S. P. W. Wijnands, J. L. J. van Dongen, L. Brunsveld, P. Y. W. Dankers, Chem. Commun. 2015, 51, 3147-3150.

[22] C. Hou, J. Li, L. Zhao, W. Zhang, Q. Luo, Z. Dong, J. Xu, J. Liu, Angew. Chem. Int. Ed. 2013, 52, 5590-5593.

[23] X. Tan, L. Yang, Y. Liu, Z. Huang, H. Yang, Z. Wang, X. Zhang, Polym. Chem. 2013, 4, 5378-5381.

[24] W. Xu, Q. Song, J.-F. Xu, M. J. Serpe, X. Zhang, ACS Appl. Mater. Interfaces 2017, 9, 11368-11372.

[25] M. J. Rowland, C. C. Parkins, J. H. McAbee, A. K. Kolb, R. Hein, X. J. Loh, C. Watts, O. A. Scherman, Biomaterials 2018, 179, 199208.

[26] D. T. Dang, H. D. Nguyen, M. Merkx, L. Brunsveld, Angew. Chem. Int. Ed. 2013, 52, 2915-2919.

[27] P. J. de Vink, J. M. Briels, T. Schrader, L.-G. Milroy, L. Brunsveld, C. Ottmann, Angew. Chem. Int. Ed. 2017, 56, 8998-9002.

[28] Z. Huang, X. Chen, G. Wu, P. Metrangolo, D. Whitaker, J. A. McCune, O. A. Scherman, J. Am. Chem. Soc. 2020, 142, 7356-7361.

[29] A. Kataki-Anastasakou, J. C. Axtell, S. Hernandez, R. M. Dziedzic, G. J. Balaich, A. L. Rheingold, A. M. Spokoyny, E. M. Sletten, J. Am. Chem. Soc. 2020, 142, 20513-20518.

[30] Z. Huang, K. Qin, G. Deng, G. Wu, Y. Bai, J.-F. Xu, Z. Wang, Z. Yu, O. A. Scherman, X. Zhang, Langmuir 2016, 32, 12352-12360.

[31] F. Biedermann, M. Vendruscolo, O. A. Scherman, A. De Simone, W. M. Nau, J. Am. Chem. Soc. 2013, 135, 14879-14888. 
